\title{
Fredholm Determinant for Piecewise Linear Transformations on a Plane
}

\author{
Makoto MORI
}

Nihon University

(Communicated by K. Katayama)

\begin{abstract}
Certain piecewise linear expanding maps on a finite union of polygons in $\mathbf{R}^{2}$ are considered. The Perron-Frobenius operator associated with a map is considered on a locally convex linear space which is an extension of the space of bounded variation functions, and the spectrum of it is determined by Fredholm matrices. New signed symbolic dynamics are defined by using screens, and the Fredholm matrices are constructed by renewal equations on this signed symbolic dynamics.
\end{abstract}

\section{Introduction.}

We will consider a class of piecewise linear expanding mappings $F$ from a subset of $\mathbf{R}^{2}$ into itself. The Perron-Frobenius operator $P$ from $L^{1}$ into itself corresponding to a mapping $F$ is defined by

$$
\int f(x) g(F(x)) d x=\int P f(x) g(x) d x \quad\left(g \in L^{\infty}\right) .
$$

We already knew in one-dimensional cases that the ergodic properties of dynamical systems are characterized by the spectrum of the Perron-Frobenius operators restricted to $B V$, the set of functions with bounded variation, and we have proved that the eigenvalues of the Perron-Frobenius operator $P$ outside of some disc are determined by the Fredholm matrix $\Phi(z)$, and at the same time the Ruelle-Artin-Mazur zeta function is expressed by the reciprocal of $\operatorname{det}(I-\Phi(z))([10],[11])$.

In this article, we will extend the notion of signed symbolic dynamics which we used in [10] and [11], and as new signs we will introduce screens. On this new signed symbolic dynamics, we will construct renewal equations, and by these renewal equations we can define Fredholm matrices $\left\{\Phi_{n}(z)\right\}$.

Let

$$
\xi=\liminf _{n \rightarrow \infty} \underset{x \in I}{\operatorname{essinf}} \frac{1}{n} \log \left|\operatorname{det} D\left(F^{n}\right)(x)\right|,
$$

Received May 23, 1997 
where $D\left(F^{n}\right)$ is the jacobian matrix of $F^{n}$. We call $\xi$ a lower Lyapunov number, which is an essential infimum of the sum of Lyapunov exponents. We call that $F$ is expanding if

$$
\underline{\xi}=\liminf _{n \rightarrow \infty} \frac{1}{n} \underset{x \in I}{\operatorname{essinf}} \log \left[\text { minimum of } \mid \text { the eigenvalue of } D\left(F^{n}\right)(x) \mid\right]>0 .
$$

Our aim in this article is to construct Fredholm matrices $\Phi_{n}(z)$ and prove (cf. [1], [4], [6]):

THEOREM A. Suppose that $F$ is piecewise linear and expanding from a finite union of bounded convex polygons I into itself, and assume that the lower Lyapunov number $\xi>0$ and satisfies $\xi>v$. Take arbitrary $\varepsilon>0$, then there exists $n_{0}>0$ such that for any $n \geq n_{0}$ in the disk $|z|<e^{\xi-v-\varepsilon}, \operatorname{det}\left(I-\Phi_{n}(z)\right)$ is analytic, and moreover $z^{-1}$ belongs to the spectrum of the Perron-Frobenius operator $P$ restricted to $\mathscr{B}$ if and only if

$$
\operatorname{det}\left(I-\Phi_{n}(z)\right)=0 \text {. }
$$

We will also prove (cf. [6], [7]):

THEOREM B. Assume that $F$ satisfies the same conditions as in Theorem A. Then the eigenfunctions of $P$ on $L^{1}$ associated with eigenvalues of modulus 1 belong to $\mathscr{B}$.

We will define the constant $v$ and give an example which satisfies $\xi>v$ in $\$ 5$. We will also define the space $\mathscr{B}$ in $\S 5$. The space $\mathscr{B}=\mathscr{B}(F)$ is a locally convex space with norms $\|\cdot\|_{v^{\prime}}\left(v^{\prime}>v\right)$, and $z^{-1}$ belongs to the spectrum of $P$ if there exists no continuous $(I-z P)^{-1}$ with norm $\|\cdot\|_{v^{\prime}}$ for all $v^{\prime}>v$.

In $\S 2, \S 3$ and $\S 4$, we will give formal discussions on generating functions and renewal equations of them. In $\S 5$, we will consider a family of functions $\mathscr{B}$ and study the eigenvalues of the Perron-Frobenius operator $\boldsymbol{P}$ on $\mathscr{B}$.

We will study higher dimensional cases in [13].

\section{Preliminaries.}

In one-dimensional cases, we introduced the signed symbolic dynamics, which represents the endpoints of the subintervals of monotonicity. It made us possible to construct a renewal equation by tracing the orbits of the endpoints separately. Now in 2-dimensional cases, we will introduce the notion of screens of polygons and trace the orbits of screens to construct renewal equations.

We denote by $J^{c l}$ and $J^{o}$ the closure and the interior of a set $J$, and the boundary $\Delta J=J^{c l} \backslash J^{o}$. Let $J$ be a bounded convex polygon for which $J^{c l}$ is homeomorphic to the unit disk. We denote by $D_{0}^{J}$ and $D_{1}^{J}$ the set of vertices (0-dimensional faces) and the set of edges (1-dimensional faces) of $J$, respectively. Set $D^{J}=D_{0}^{J} \cup D_{1}^{J}$. We call a set of lines $\pi_{1}, \cdots, \pi_{m}$ lines which determine $J$, when $J$ is the intersection of half 
spaces defined by $\pi_{1}, \cdots, \pi_{m}$. For each vertex $\partial \in D_{0}^{J}$, we call a pair of lines $\pi_{i}, \pi_{j}$ $(1 \leq i, j \leq m)$ determines $\partial$ when their intersection equals $\partial$, and for each edge $\partial \in D_{1}^{J}$, we call a line $\pi_{i}(1 \leq i \leq m)$ determines $\partial$ when $\pi_{i} \cap \Delta J$ equals $\partial$.

Let $\mathscr{A}$ be a finite set of symbols, and $\langle a\rangle(a \in \mathscr{A})$ be bounded convex polygons mutually disjoint and each $\langle a\rangle^{c l}$ is homeomorphic to the unit disk. Set $I=\bigcup_{a \in A}\langle a\rangle$. We consider a transformation $F: I \rightarrow I$, for which the restriction $F^{a}$ of $F$ to $\langle a\rangle$ is an affine map, that is, there exists a vector $p^{a}$ and a matrix $M^{a}$ and

$$
F^{a}(x)=p^{a}+M^{a} x
$$

As we assumed that $F$ is expanding, it follows $\operatorname{det} M^{a} \neq 0$ for all $a \in \mathscr{A}$. We denote by $F^{i}$ the $i$-th iteration of $F$ :

$$
F^{i}(x)= \begin{cases}x & i=0, \\ F\left(F^{i-1}(x)\right) & i \geq 1 .\end{cases}
$$

REMARK 1. A transformation $F$ need not to be continuous on $\Delta\langle a\rangle(a \in \mathscr{A})$. However, whether the boundaries $\Delta\langle a\rangle$ of $a \in \mathscr{A}$ belongs to $\langle a\rangle$ or not does not play essential role in this article. Therefore, to avoid complicated notations, we usually ignore them.

REMARK 2. In this article, we only treat polygons which are bounded convex and homeomorphic to the unit disk. Hence, we simply call them polygons.

As in one-dimensional cases, we use the notation such as words, shift and expansions, etc. We will summarize them here.

A finite sequence of symbols $w=a_{1} \cdots a_{n}$ is called a word and we set

1. $|w|=n$ (the length of $w$ ), (for the empty word $\varepsilon$, we put $|\varepsilon|=0$ ),

2. $\langle w\rangle= \begin{cases}\bigcap_{i=1}^{n}\left(F^{i-1}\right)^{-1}\left(\left\langle a_{i}\right\rangle\right) & \text { if } w \neq \varepsilon, \\ I & \text { if } w=\varepsilon \text { (for notational convenience), }\end{cases}$

3. $w[k]=a_{k}$ for $1 \leq k \leq n$,

4. $w[k, l]=a_{k} \cdots a_{l}$ for $1 \leq k \leq l \leq n$,

5. $\theta w=a_{2} \cdots a_{n}$ (shift),

6. $\quad F^{w}=F^{a_{n}} \cdots F^{a_{1}},\left(F^{\varnothing}=\right.$ identity map $)$.

We denote by $\mathscr{W}$ the set of words $w$ with $\langle w\rangle \neq \varnothing$. We call a word $w \in \mathscr{W}$ admissible. Note that the empty word $\varepsilon \in \mathscr{W}$.

Remark 3. We assumed $\operatorname{det} M^{a} \neq 0$ for all $a \in \mathscr{A}$. Therefore for each $a \in \mathscr{A}$, the domain of the mapping $F^{a}$ can be extended naturally to $\mathbf{R}^{2}$ and we can define $\left(F^{a}\right)^{-1}$ from $I$ into $\mathbf{R}^{2}$. Therefore, for any $x \in I$, we can define $\left(F^{a}\right)^{-1}(x)=a x \in \mathbf{R}^{2}$. In a same way, for any word $w \in \mathscr{W}$, we can define $w x \in \mathbf{R}^{2}$ by $w x=\left(F^{w}\right)^{-1}(x)$. If $w x \in\langle w\rangle$, then, of course, $F^{|w|}(w x)=x$. For this case, we call that $w x$ exists. For a fixed $x \in I$, the notation 
$\sum_{w: \exists w x}$ means the sum over all words $w$ for which $w x$ exists.

We considered, for one-dimensional cases, the symbolic dynamics with the set of signs $\{+,-\}$. Now in 2-dimensional cases, we use the set of faces to construct renewal equations. For a vertex $\partial \in D_{0}^{J}$, let $\pi, \pi^{\prime}$ be the lines which satisfy $\Delta J \cap\left(\pi \cap \pi^{\prime}\right)=\partial$, and $\Delta J \cap \pi, \Delta J \cap \pi^{\prime} \in D_{1}^{J}$ ( $\pi$ and $\pi^{\prime}$ determine $\partial$ ). Then $\mathbf{R}^{2}$ is divided into four regions by the lines $\pi$ and $\pi^{\prime}$. There exists a unique region which contains $J^{o}$, and we call it the interior region determined by $\partial$. We denote its boundary by $J^{\partial}$ (a union of two half lines which start from $\partial$ ) and call it the screen of the vertex $\partial$. Also for an edge $\partial \in D_{1}^{J}$, let $\pi$ be the line which satisfies $\Delta J \cap \pi=\partial$, $\left(\partial\right.$ is determined by $\pi$ ). Then $\mathbf{R}^{2}$ is divided into two regions by the line $\pi$. We also call a unique region which contains $J^{o}$ by the interior region determined by $\partial$. The line $\pi$ is denoted by $J^{\partial}$ and called the screen of the edge $\partial$. Dividing $\mathbf{R}^{2}$ into two regions, inside and outside by $J^{\partial}$, put

$$
\sigma\left(J^{\partial}, x\right)= \begin{cases}+1 & \text { if } x \text { belongs to the interior region determined by } \partial, \\ -1 & \text { otherwise }\end{cases}
$$

Note that as we mentioned before, we ignore the case $x \in J^{\partial}$, because it is unessential.

For a word $w$, we can naturally define $\sigma\left(F^{w} J^{\partial}, x\right)=\sigma\left(J^{\partial}, w x\right)$. Note that $\sigma\left(F^{w} J^{\partial}, x\right)$ is defined for all $x \in \mathbf{R}^{2}$ and for all word $w$ even if $w x$ does not exist (that is, even if $\langle w\rangle=\varnothing$ or if there exists no $y \in\langle w\rangle$ such that $\left.F^{|w|}(y)=x\right)$. This is one of the advantage to use the signed symbolic dynamics.

For readers' convenience, we will give examples of screens (Figure 1). Let $J$ be a rectangle in $\mathbf{R}^{2}$. Then there exist four 1-dimensional faces (say them $l, r, u, d$ (left, right, and so on)) and also four 0-dimensional faces ( $l u, l d, r u, r d$, (left-upper and so on)), that is,

$$
D_{0}^{J}=\{l u, l d, r u, r d\}, \quad D_{1}^{J}=\{l, r, u, d\}, \quad D^{J}=D_{0}^{J} \cup D_{1}^{J} .
$$

Set

$$
s_{k}=(-1)^{k} / 2(k=0,1), \quad s_{2}=1 .
$$

We also use the notation

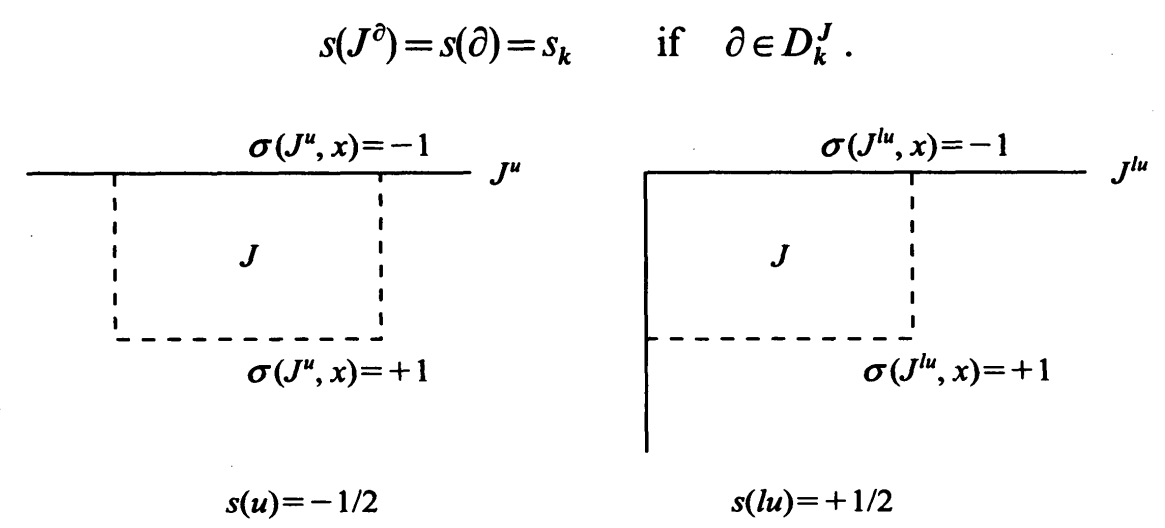

FIGURE 1 
Even if two screens $J^{\partial}$ and $K^{\partial^{\prime}}$ for polygons $J$ and $K$ are equal as sets, if $J \neq K$ we consider them as different screens.

For a word $w \in \mathscr{W}$, we denote $w^{\partial}$ instead of $\langle w\rangle^{\partial}$, and we denote

$$
\tilde{\mathscr{A}}=\left\{a^{\partial}: a \in \mathscr{A}, \partial \in D^{\langle a\rangle}\right\} \text {. }
$$

For a face $\partial$, we call that a face $\partial^{\prime}$ is contained in $\partial$ if the set of the lines which determine $\partial^{\prime}$ contains all the lines which determine $\partial$, and we denote $\partial^{\prime} \subset \partial$. Here we consider $\partial \subset \partial$.

Now we get a simple but key lemma.

LEMMA 2.1. Let $J$ be a polygon.

(i) Take an edge $\partial$ and a vertex $\partial_{0} \subset \partial$ of $J$. Let $U$ be a neighborhood of $\partial_{0}$ sufficiently small. Then

$$
\sum_{\substack{\partial^{\prime} \subset \partial \\ \partial^{\prime} \neq \partial_{0}}} s\left(\partial^{\prime}\right) \sigma\left(J^{\partial^{\prime}}, x\right)=0
$$

(ii) Let $M$ be a set of edges of a polygon $J$ such that $\bigcup_{\partial \in M} \partial$ is homeomorphic to a closed interval. Denote by $\partial_{s}, \partial_{e} \in D_{0}^{J}$ the endpoints of $M$, that is, $M \backslash\left(\partial_{s} \cup \partial_{e}\right)$ is homeomorphic to an open interval. Then

$$
\sum_{\substack{\partial^{\prime} \subset \exists \partial \in M \\ \partial^{\prime} \neq \partial_{s}, \partial_{e}}} s\left(\partial^{\prime}\right)=-\frac{1}{2},
$$

where the sum is over all $\partial^{\prime} \in D^{J}$ for which there exists $\partial \in M$ such that $\partial^{\prime} \subset \partial$.

(iii) Denote by $1_{J}$ the indicator function of a convex polygon $J$. Then we get

$$
\sum_{\partial \in D^{J}} s(\partial) \sigma\left(J^{\partial}, x\right)+s_{2}=1_{J}(x) \quad \text { a.e. } x .
$$

Proof. (i) Let $\pi$ be a line which determine $\partial$, and $\partial_{1}$ be endpoint of $\partial$ different from $\partial_{0}$. Let $\pi$ and $\pi^{\prime}$ be lines which determine $\partial_{1}$. Take $U$ sufficiently small such that $U$ does not cross $\pi^{\prime}$. Then if $x \in U$ belongs to the interior of the screen $J^{\partial}$, it also belongs to the interior of the screen $J^{\partial_{1}}$. Therefore $\sigma\left(J^{\partial}, x\right)=\sigma\left(J^{\partial_{1}}, x\right)=1$. Since $s(\partial)=-1 / 2$ and $s\left(\partial_{1}\right)=+1 / 2$, this proves (i) for this case. The proof is just the same when $x$ belongs to the outside of the screen $J^{\partial}$.

(ii) Now put $M=\left\{\partial_{1}, \cdots, \partial_{k}\right\}\left(\partial_{i} \in D_{1}^{J}\right)$ such that $\partial_{i} \cap \partial_{i+1} \neq \varnothing(i=1, \cdots, k-1)$. Then there exists $k$ edges and $(k-1)$ vertices except $\partial_{s}, \partial_{e}$. This shows (ii).

(iii) In a similar arguments as in (i), fix a vertex $\partial_{0}$ of $J$ and take a sufficiently small neighborhood $U$ of $\partial_{0}$. Let $\partial_{1}$ and $\partial_{2}$ be the edges of $J$ such that $\partial_{1} \cap \partial_{2}=\partial_{0}$. Denote by $M$ the union of all the edges $\partial \in D_{1}^{J}$ except $\partial_{1}$ and $\partial_{2}$. Then, $M$ satisfies the assumption of (ii). From (ii), for $x \in U$

$$
\sum s(\partial) \sigma\left(J^{\partial}, x\right)=-1 / 2
$$

where the sum is over $\partial \in D^{J}$ except the faces contained in $\partial_{1}$ and $\partial_{2}$. The rest of faces, 


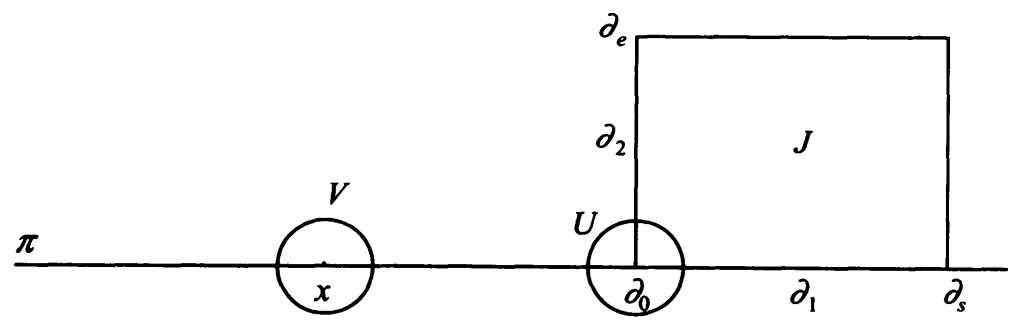

FIGURE 2

$\partial_{0}, \partial_{1}, \partial_{2}$ and $\partial_{s}, \partial_{e}$ give $+1 / 2$ for a point $x \in U \cap J$, and $-1 / 2$ for a point $x \in U \cap J^{c}$ $(x \notin \Delta J)$. This proves (3) for $x \in U$. When we move $x$ along a curve, it will change value when $x$ crosses a line $\pi$ which generates some face of $J$. As we assume $J$ convex, the equation (3) also holds for every point in $J^{o}$. Let $x \notin J^{c l}$ be a point on $\pi$ which generates some face of $J$, and we will consider a sufficiently small neighborhood $V$ of $x$. Note that only two screens corresponding to one vertex and one edge, give different values to $y \in V$ depending on the side of $\pi$ where $y$ belongs. But their influence to the right hand term of (3) cancells, because $s(\partial)=+1 / 2$ (or $-1 / 2)$ for a vertex (an edge), respectively. This proves (iii) (cf. Figure 2).

Now we define generating functions to construct a renewal equation. We will discuss the radius of convergence of generating functions in $\$ 5$, and in this section we consider them as formal power series. Put for a polygon $J$ and $g \in L^{\infty}$

$$
s_{g}^{J}(z)=\int d x g(x) \sum_{\substack{w: \exists w x \in J \\ w \in W}} z^{|w|} \eta(w)
$$

and for $J \subset\langle a\rangle(a \in \mathscr{A})$ and $\partial \in D^{J}$

$$
s_{g}^{J^{\partial}}(z)=\int d x g(x) \sum_{\exists \theta w x,\langle w[1]\rangle \supset\langle a\rangle} z^{|w|} \eta(w) \sigma\left(J^{\partial}, w x\right),
$$

where for a word $w=a_{1} \cdots a_{n}$

$$
\eta(w)=\prod_{i=1}^{n}\left|\operatorname{det} M^{a_{i}}\right|^{-1}
$$

Concerning the notation $\exists w x$, refer Remark 3, and for a word $w$

$$
\langle w[1]\rangle=\left\{\begin{array}{lll}
\left\langle a_{1}\right\rangle & \text { if } & w=a_{1} \cdots a_{n} \\
I & \text { if } \quad w=\varepsilon \text { (empty word) . }
\end{array}\right.
$$

REMARK 4. (1) In the definitions of $s_{g}^{J^{\partial}}(z)$ we consider words $w$ for which $\theta w x$ exist but $w x$ do not necessarily exist. This is one of the key point of this article.

(2) Noticing the fact that $w x$ is the one of the inverse image of $F^{-|w|}(x), s_{g}^{J^{o}}(z)$ can be written as a formal expression 


$$
\begin{aligned}
s_{g}^{J^{\partial}}(z) & =\int d x g(z) \sum_{\exists \theta w x,\langle w[1]\rangle \supset\langle a\rangle} z^{|w|} \eta(w) \sigma\left(J^{\partial}, w x\right) \\
& =\int_{I} d x g(x) \sigma\left(J^{\partial}, x\right)+\sum_{n=1}^{\infty} z^{n} \int d x g(x) \sum_{\exists \theta w x, w[1]=a,|w|=n} \eta(w) \sigma\left(J^{\partial}, w x\right) \\
& =\int_{I} d x g(x) \sigma\left(J^{\partial}, x\right)+\sum_{n=1}^{\infty} z^{n} \int d x g(x)\left[P^{n}\left(\sigma\left(J^{\partial}, \cdots\right)\right)\right](x) \\
& =\int d x g(x) z \eta(a)\left[(I-z P)^{-1}\left(\sigma\left(F^{a} J^{\partial}, \cdot\right)\right)\right](x) .
\end{aligned}
$$

(3) Since $P$ is contracting in $L^{1}$, in $|z|<1$ all the above series converge.

(4) Note also $s_{g}^{J}(z) \equiv 0$ for a polygon $J$ which is contained in the complement of $I$.

(5) Let $J^{\partial}$ and $K^{\partial^{\prime}}$ be screens of polygons $J \subset\langle a\rangle$ and $K \subset\langle b\rangle(a, b \in \mathscr{A})$ respectively and they are equal as sets. Then if $a=b$,

$$
s_{g}^{J^{\partial}}(z)=s_{g}^{\mathbf{K}^{\boldsymbol{\theta}^{\prime}}}(z),
$$

that is, in Figure $3, s_{g}^{a^{\partial}}(z)$ does not equal $s_{g}^{b^{\partial^{\prime}}}(z)$ unless $F^{a}=F^{b}$, but $s_{g}^{a^{\partial}}(z)=s_{g}^{J^{\partial}}(z)$. We will use this relation in the next section to construct a renewal equation.

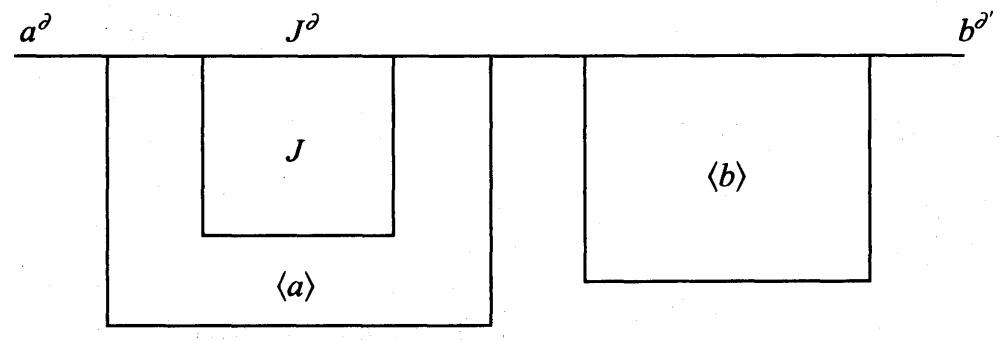

FIGURE 3

LEMMA 2.2. For a polygon $J$ such that $J \subset\langle a\rangle$ and $g \in L^{\infty}$,

$$
\begin{gathered}
s_{g}^{J}(z)=\int_{J} g(x) d x+z \eta(a) s_{g}^{F(J)}(z), \\
s_{g}^{J}(z)=s_{2} \int_{I} d x g(x)+s_{2} z \eta(a) s_{g}^{I}(z)+\sum_{\partial \in D^{J}} s(\partial) s_{g}^{J^{\partial}}(z) .
\end{gathered}
$$

Proof. Dividing the case $|w|=0$ and $|w| \geq 1$, we get the proof of (4). In a same way, dividing the case $|w|=0$ and $|w| \geq 1$,

$$
\begin{aligned}
\sum_{\partial \in D^{J}} s(\partial) s_{g}^{J^{\partial}}(z)= & \sum_{\partial \in D^{J}} \int_{I} d x g(x) s(\partial) \sigma\left(J^{\partial}, x\right) \\
& +\sum_{\partial \in D^{J}} \int d x g(x) \sum_{\exists \theta w x, w[1]=a} z^{|w|} \eta(w) s(\partial) \sigma\left(J^{\partial}, w x\right) .
\end{aligned}
$$


Here, we again use the expression stated in Remark 3. The sum $\sum_{\exists \theta w x, w[1]=a}$ denotes the sum over all words $w=a_{1} \cdots a_{n}$ such that $a_{1}=a$ and $\theta w x=a_{2} \cdots a_{n} x$ exists, that is, there exists a point $y \in I$ such that $y \in\left\langle a_{2}\right\rangle, F(y) \in\left\langle a_{3}\right\rangle, \cdots, F^{n-2}(y) \in\left\langle a_{n}\right\rangle$ and $F^{n-1}(y)=x$. Note again, we need not assume that $w x$ exists. Then by Lemma 2.1 (iii), we get

$$
\sum_{\partial \in D^{J}} \int d x g(x) s(\partial) \sigma\left(J^{\partial}, x\right)=\int d x g(x)\left(1_{J}(x)-s_{2}\right)=\int d x g(x) 1_{J}(x)-s_{2} \int d x g(x) .
$$

Taking $\theta w$ as $w$, we get

the second term of rhs. of (6)

$$
\begin{aligned}
& =z \eta(a) \int d x g(x) \sum_{w: \exists w x} z^{|w|} \eta(w) \sum_{\partial \in D^{J}} s(\partial) \sigma\left(J^{\partial}, a w x\right) \\
& =z \eta(a) \int d x g(x) \sum_{w: \exists w x} z^{|w|} \eta(w)\left[1_{J}(a w x)-s_{2}\right] \\
& =\int d x g(x) \sum_{w: \exists a w x \in J} z \eta(a) z^{|w|} \eta(w)-z \eta(a) s_{2} \int d x g(x) \sum_{w: \exists w x} z^{|w|} \eta(w) \\
& =\int d x g(x) \sum_{|w| \geq 1, \exists w x \in J} z^{|w|} \eta(w)-s_{2} z \eta(a) \int d x g(x) \sum_{w: \exists w x} z^{|w|} \eta(w) \\
& =\int d x g(x) \sum_{|w| \geq 1, \exists w x \in J} z^{|w|} \eta(w)-s_{2} z \eta(a) s_{g}^{I}(z) .
\end{aligned}
$$

Therefore combining (7) and (8), we get

$$
\text { rhs. of }(6)=s_{g}^{J}(z)-s_{2}\left[\int_{I} d x g(x)+z \eta(a) s_{g}^{I}(z)\right] \text {. }
$$

This proves (5).

COROLlaRY 2.1. We have a renewal equation of the form:

$$
s_{g}^{I}(z)=s_{2} \# \mathscr{A} \int g d x+s_{2} z\left(\sum_{a \in \mathscr{A}} \eta(a)\right) s_{g}^{I}(z)+\sum_{a \in \mathscr{A}} \sum_{\partial \in D^{\langle a\rangle}} s(\partial) s_{g}^{a^{\partial}}(z) .
$$

Since $s_{g}^{I}(z)=\sum_{a \in \mathscr{A}} s_{g}^{\langle a\rangle}$, the proof directly follows from (5).

As a formal discussion (rigorous discussion will appear in §5), the spectral problem of the Perron-Frobenius operator $P$ turns into the problem of singularities of $s_{g}^{J}(z)$, since

$$
s_{g}^{J}(z)=\int\left[(I-z P)^{-1} 1_{J}\right](x) g(x) d x .
$$

To solve this problem, we will construct renewal equations. However, as we stated in [10] even for one-dimensional piecewise linear transformations, it is almost impossible 
to construct renewal equations for $s_{g}^{J}(z)$, because we must trace the orbits of $\Delta\langle a\rangle$ at a time. But by Lemma 2.2, it is sufficient to construct renewal equations for $s_{g}^{J^{\partial}}(z)$.

\section{Renewal equations.}

Renewal equations are well known notion in the theory of Markov processes (cf. for example [3]). First, we will show how to use it to our discussion in a very simple example.

EXAMPLE 1. Let us explain a renewal equation by a simple Markov case (cf. Figure 4). Here $\langle a\rangle=\triangle A C D,\langle b\rangle=\triangle B C D$ and $I=\langle a\rangle \cup\langle b\rangle$ (we ignore the segments $C D$, because it does not affect the result), and

$$
\begin{array}{lll}
F^{a}(A)=A, & F^{a}(D)=C, & F^{a}(C)=B, \\
F^{b}(C)=A, & F^{b}(D)=D, & F^{b}(B)=C .
\end{array}
$$

If $A B>A C>A D>C D$ and $A C>B C$, then this mapping is expanding.

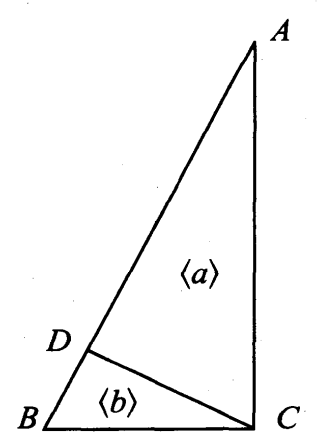

FIGURE 4

Now, since $F^{a}(\langle a\rangle)=\langle a\rangle \cup\langle b\rangle$,

$$
\begin{aligned}
s_{g}^{\langle a\rangle}(z) & =\sum_{n=0}^{\infty} z^{n} \int 1_{\langle a\rangle}(x) g\left(F^{n}(x)\right) d x \\
& =\int_{\langle a\rangle} g(x) d x+\sum_{n=1}^{\infty} z^{n} \int 1_{\langle a\rangle}(x) g\left(F^{n}(x)\right) d x \\
& =\int_{\langle a\rangle} g(x) d x+z \eta(a)\left(s_{g}^{\langle a\rangle}(z)+s_{g}^{\langle b\rangle}(z)\right),
\end{aligned}
$$

and in a similar way, since $F^{b}(\langle b\rangle)=\langle a\rangle$,

$$
s_{g}^{\langle b\rangle}(z)=\int_{\langle b\rangle} g(x) d x+z \eta(b) s_{g}^{\langle a\rangle}(z) .
$$

This is a renewal equation, and we get 


$$
\left(\begin{array}{l}
s_{g}^{\langle a\rangle}(z) \\
s_{g}^{\langle b\rangle}(z)
\end{array}\right)=\left(\begin{array}{cc}
1-z \eta(a) & -z \eta(a) \\
-z \eta(b) & 1
\end{array}\right)^{-1}\left(\begin{array}{l}
\int_{\langle a\rangle} g(x) d x \\
\int_{\langle b\rangle} g(x) d x
\end{array}\right) .
$$

From this renewal equation, we can solve the problem of singularities of $s_{g}^{\langle a\rangle}(z)$ and $s_{g}^{\langle b\rangle}(z)$ by solving

$$
\left|\begin{array}{cc}
1-z \eta(a) & -z \eta(a) \\
-z \eta(b) & 1
\end{array}\right|=0
$$

But as in one-dimensional cases, for non-Markov transformations it is almost impossible to construct a renewal equation for $s_{g}^{\langle a\rangle}(z)(a \in \mathscr{A})$.

Notation 1. For symbols $a$ and $b(a, b \in \mathscr{A})$, we say that a screen $\tilde{J}$ of a polygon $J$ crosses $a b$ if $J \subset\langle a\rangle$ and $F^{a}(\widetilde{J}) \cap\langle b\rangle \neq \varnothing$ (cf. Figure 5, the definitions of $\Delta_{0}, \Delta_{1}$ and $\Delta_{2}$ will be given afterwards). Set

$$
\langle a b, \tilde{J}\rangle= \begin{cases}\left\{x \in\langle b\rangle: \sigma\left(F^{a}(\tilde{J}), x\right)=+1\right\} & \text { if } \tilde{J} \text { crosses } a b, \\ \varnothing & \text { otherwise }\end{cases}
$$

A screen $\langle a b, \tilde{J}\rangle^{\partial}$ with a face $\partial \in D^{\left\langle a b, J^{\prime}\right\rangle}$ such that $\langle a b, \tilde{J}\rangle^{\partial}$ and $b^{\partial^{\prime}}$ are different as sets for any $\partial^{\prime} \in D^{\langle b\rangle}$ is called a new screen generated by $F^{a}(\tilde{J})$ in $\langle b\rangle$, and we denote by $N e w\langle a b, \tilde{J}\rangle$ the set of new screens of $\langle a b, \tilde{J}\rangle$.
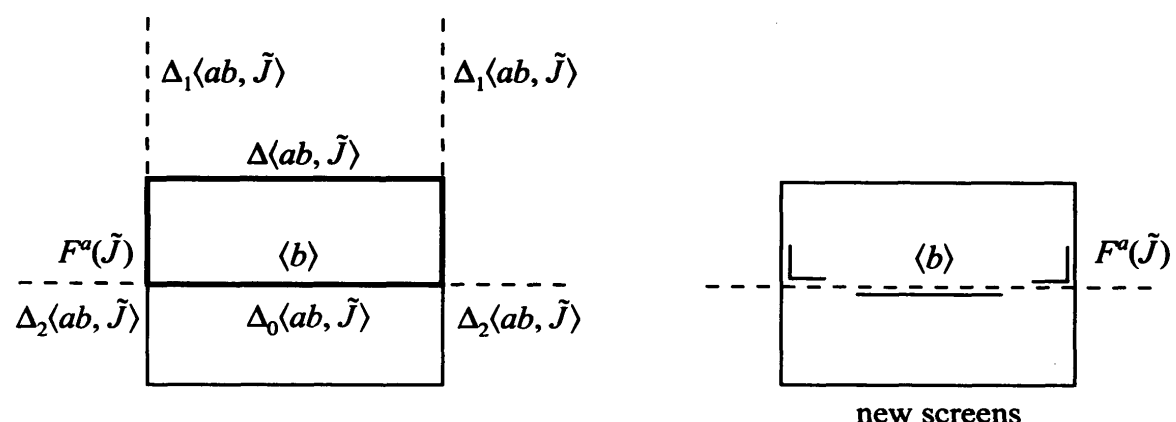

Figure 5

REMARK 5. Note that if $K^{\partial}=b^{\partial^{\prime}}$ and $K \subset\langle b\rangle$, then $s_{g}^{K^{\partial}}(z)=s_{g}^{b^{\partial^{\prime}}}(z)$.

Before the next step to describe the notations, we will mention several lemmas.

LEMMA 3.1. For a screen J of a polygon $J \subset\langle a\rangle$ for some $a \in \mathscr{A}$ and $g \in L^{\infty}$, we get:

(i) First renewal equation:

$$
s_{g}^{J}(z)=\int d x g(x) \sigma(\tilde{J}, x)+z \eta(a)\left\{\sum_{b \in \mathscr{A}} s_{g}^{\langle b\rangle}(z) \sigma_{0}\left(F^{a}(\tilde{J}), b\right)+2 \sum_{b \in \mathscr{A}} s_{g}^{\langle a b, \tilde{J}\rangle}(z)\right\}
$$

where 


$$
\sigma_{0}\left(F^{a}(\widetilde{J}), b\right)= \begin{cases}+1 & \text { if } \sigma(\widetilde{J}, a x)=+1 \text { holds for a.e. } x \in\langle b\rangle, \\ -1 & \text { otherwise } .\end{cases}
$$

(ii) Second renewal equation:

$$
\begin{aligned}
s_{g}^{\mathcal{J}}(z)= & \tilde{\chi}_{g}^{\mathcal{J}}(z)+\sum_{\tilde{b} \in \tilde{A}} z \phi^{\mathcal{J}}(\tilde{b}) s_{g}^{\tilde{b}}(z)+z^{2} \phi^{\widetilde{J}}(I) s_{g}^{I}(z) \\
& +2 z \eta(a) \sum_{b \in \mathscr{A}} \sum_{\tilde{K} \in N e w\langle a b, \tilde{J}\rangle} s(\widetilde{K}) s_{g}^{\tilde{K}}(z),
\end{aligned}
$$

where

$$
\begin{gathered}
\tilde{\chi}_{g}^{\tilde{J}}(z)=\int g(x) \sigma(\tilde{J}, x) d x+z s_{2} \eta(a) \sum_{b \in \mathscr{A}} \sigma_{*}\left(F^{a}(\widetilde{J}), b\right) \int g(x) d x, \\
\phi^{\tilde{J}}(\tilde{b})=s(\tilde{b}) \eta(a) \sigma_{*}\left(F^{a}(\tilde{J}), \tilde{b}\right), \quad \phi^{\tilde{J}}(I)=\eta(a) s_{2} \sum_{b \in \mathscr{A}} \sigma_{*}\left(F^{a}(\widetilde{J}), b\right) \eta(b),
\end{gathered}
$$

and

$$
\begin{gathered}
\sigma_{*}\left(F^{a}(\widetilde{J}), b\right)= \begin{cases}-1 & \text { if } \sigma\left(F^{a}(\tilde{J}), x\right)=-1 \text { holds for a.e. } x \in\langle b\rangle, \\
+1 & \text { otherwise },\end{cases} \\
\sigma_{*}\left(F^{a}(\widetilde{J}), \tilde{b}\right)= \begin{cases}+1 & \text { if } \sigma\left(F^{a}(\widetilde{J}), x\right)=+1 \text { for all } x \in\langle b\rangle, \\
& \text { or if } \widetilde{J} \text { crosses ab and } \tilde{b}=\langle a b, \widetilde{J}\rangle^{\partial} \\
-1 & \text { for some } \partial \in D^{\langle a b, \tilde{J}\rangle},\end{cases} \\
\text { otherwise. }
\end{gathered}
$$

Proof. In Figure 6, we show the values of $\sigma_{0}$ and $\sigma_{*}$ for simple cases. Taking $\theta w$ as $w$, the left hand side of the definition of $s_{g}^{J}(z)$ for $|w|>0$ becomes:

$$
\begin{aligned}
\int d x g(x) \sum_{\exists \theta w x, w[1]=a} z^{|w|} \eta(w) \sigma(\tilde{J}, w x) \\
=z \eta(a) \sum_{b \in \mathscr{A}} \int d x g(x) \sum_{\exists w x \in\langle b\rangle} z^{|w|} \eta(w) \sigma\left(F^{a}(\widetilde{J}), w x\right) .
\end{aligned}
$$

If $\langle b\rangle$ does not intersect $F^{a}(\widetilde{J}), \sigma\left(F^{a}(\widetilde{J}), w x\right)$ is independent of $w x \in\langle b\rangle$ and equals

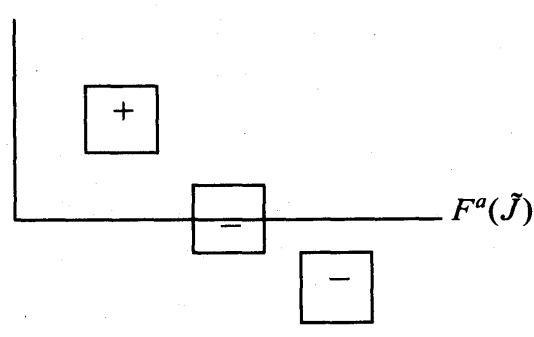

The values of $\sigma_{0}\left(F^{a}(\tilde{J}), b\right)$ for various rectangles $\langle b\rangle$

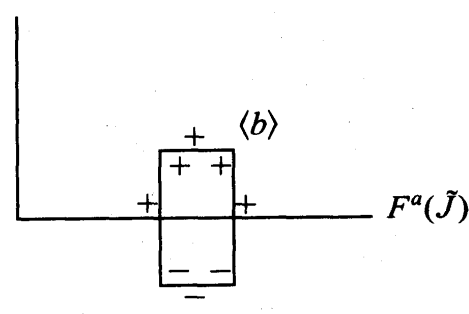

The values of $\sigma_{*}\left(F^{a}(\tilde{J}), b^{\partial}\right)$ for each face $\partial$ of $\langle b\rangle$

Figure 6 
$\sigma_{0}\left(F^{a}(\widetilde{J}), b\right)$. If $F^{a}(\widetilde{J})$ intersects $\langle b\rangle$, we take

$$
\sigma\left(F^{a}(\widetilde{J}), w x\right)=-1+2 \times 1_{\langle a b, J\rangle}(w x)=\sigma_{0}\left(F^{a}(\widetilde{J}), w x\right)+2 \times 1_{\langle a b, J\rangle}(w x),
$$

where $1_{J}$ is the indicator function of a set $J$. This leads to the proof of (i), and the proof of (ii) follows from (i) using (5):

$$
\begin{aligned}
s_{g}^{\tilde{J}}(z)= & \int d x g(x) \sigma(\tilde{J}, x) \\
& +z \eta(a) \sum_{b \in \mathscr{A}} \sigma_{0}\left(F^{a}(\tilde{J}), b\right)\left[s_{2} \int d x g(x)+s_{2} z \eta(b) s_{g}^{I}(z)+\sum_{\partial \in D^{\langle b\rangle}} s(\partial) s_{g}^{b^{\partial}}(z)\right] \\
& +2 z \eta(a) \sum_{\substack{b \in \mathscr{A} \\
F^{a}\left(\boldsymbol{J}^{\prime} \cap\langle b\rangle \neq \varnothing\right.}}\left[s_{2} \int d x g(x)+s_{2} z \eta(b) s_{g}^{I}(z)+\sum_{\partial \in D^{\langle a b . J\rangle}} s(\partial) s_{g}^{\left\langle a b, J^{\partial}(z)\right] .}\right.
\end{aligned}
$$

Decomposing the screens of $\langle a b, \tilde{J}\rangle$ into new and old screens, we get using Remark 5(1)

$$
\begin{aligned}
s_{g}^{J}(z)= & \int d x g(x) \sigma(\tilde{J}, x)+z \eta(a) s_{2} \sum_{b \in \mathscr{A}} \sigma_{*}\left(F^{a}(\tilde{J}), b\right) \int d x g(x) \\
& +z^{2} \eta(a) s_{2}\left(\sum_{b \in \mathscr{A}} \sigma_{*}\left(F^{a}(\widetilde{J}), b\right) \eta(b)\right) s_{g}^{I}(z)+z \eta(a) \sum_{\tilde{b} \in \tilde{A}} \sigma_{*}\left(F^{a}(\tilde{J}), \tilde{b}\right) s(\tilde{b}) s_{g}^{\tilde{\delta}}(z) \\
& +2 z \eta(a) \sum_{b \in \mathscr{A}} \sum_{\tilde{K} \in N e w\langle a b, J} s(\tilde{K}) s_{g}^{\tilde{K}}(z) .
\end{aligned}
$$

This proves (ii).

LEMMA 3.2. Let $\Omega$ be a domain in which $s_{g}^{J}(z)$ and $s_{g}^{\tilde{K}}(z)$ are meromorphic for all polygons $J$ and screens $\tilde{K}$. Then, for $z_{0} \in \Omega$, the following two conditions are equivalent:

1. There exists $a \in \mathscr{A}$ and a polygon $J \subset\langle a\rangle$ such that $s_{g}^{J}(z)$ has a singularity at $z_{0} \in \Omega$.

2. There exists $b \in \mathscr{A}$, a polygon $K \subset\langle b\rangle$ and $\partial \in D^{k}$ such that $s_{g}^{K^{\partial}}(z)$ has a singularity at $z_{0} \in \Omega$, or $s_{g}^{I}(z)$ has a singularity at $z_{0} \in \Omega$.

Proof. First note that if $s_{g}^{I}(z)$ has singularity at $z_{0}$, then there exists $a \in \mathscr{A}$ for which $s_{g}^{\langle a\rangle}(z)$ has singularity at $z_{0}$ because $s_{g}^{I}(z)=\sum_{a \in \mathscr{A}} s_{g}^{\langle a\rangle}(z)$.

Suppose now that $s_{g}^{J}(z)$ has singularity at $z_{0}$, then by Lemma $2.2 s_{g}^{I}(z)$ or $s_{g}^{J^{j}}(z)$ must have a singularity at $z_{0}$ for some $\partial \in D^{J}$. On the contrary, if $s_{g}^{\mathcal{J}}(z)$ has a singularity at $z_{0}$, then by (10) $s_{g}^{\langle a\rangle}(z)$ or $s_{g}^{\left\langle a b, J^{\prime}\right\rangle}(z)$ for some $a, b \in \mathscr{A}$ must have a singularity at $z_{0}$.

From this lemma, to solve the spectrum problem of the Perron-Frobenius operator, we need to solve the problem of singularities of $s_{g}^{J^{\partial}}(z)$ and $s_{g}^{I}(z)$. To solve this problem of singularities, we will construct renewal equations of $s_{g}^{J^{b}}(z)$ applying second renewal equation (11) to new screens repeatedly. Though the construction is simple, however, to get renewal equations which have sufficiently large radius of convergence, we need technical and tedious calculations, therefore it is not at all easy to understand. To make 
the discussion a little bit clear, we will divide the construction of renewal equations into several steps.

First, we will begin with the second step of the inductive definition.

Notation 2. Let $w=a_{1} \cdots a_{n}$ be a word of length $n \geq 2$ and $b \in \mathscr{A}$. We call a screen $\widetilde{J}$ of a polygon $J$ crosses $w b$ if

$$
\begin{aligned}
& J \subset\left\langle a_{1}\right\rangle, \\
& F^{a_{i}}\left(\Delta_{0}\langle w[1, i], \tilde{J}\rangle\right) \cap\left\langle a_{i+1}\right\rangle \neq \varnothing \quad(1 \leq i \leq n-1), \\
& F^{a_{n}}(\tilde{K}) \cap\langle b\rangle \neq \varnothing,
\end{aligned}
$$

for some $\tilde{K} \in N e w\langle w, \tilde{J}\rangle$, where we define inductively the sets $\Delta_{0}\langle w b, \tilde{J}\rangle,\langle w b, \widetilde{J}\rangle$ and the new screens $N e w\langle w b, \widetilde{J}\rangle$ generated by $F^{w}(\widetilde{J})$ in $\langle b\rangle$ as follows:

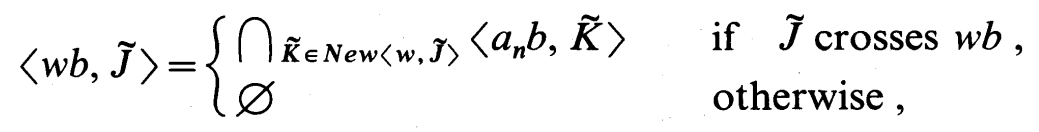

$$
\begin{aligned}
& N e w\langle w b, \tilde{J}\rangle= \begin{cases}\left\{\langle w b, \tilde{J}\rangle^{\partial}:\langle w b, \tilde{J}\rangle^{\partial} \neq b^{\partial^{\prime}}, \forall \partial^{\prime} \in D^{\langle b\rangle}\right\} & \text { if } \tilde{J} \text { crosses } w b, \\
\varnothing & \text { otherwise . }\end{cases}
\end{aligned}
$$

Note that the set $\langle w b, \widetilde{J}\rangle$ for $|w|=1$ is already defined in Notation 1 . Set

$$
\Delta_{0}\langle w b, \tilde{J}\rangle=\left(F^{w}(\tilde{J}) \cap \Delta\langle w b, \tilde{J}\rangle\right) \backslash \Delta\langle b\rangle,
$$

$\Delta_{2}\langle w b, \tilde{J}\rangle$ is the union of $\langle w b, \tilde{J}\rangle^{\partial} \backslash\left(F^{w}(\tilde{J}) \cap\langle b\rangle\right)$ such that $\langle w b, \tilde{J}\rangle^{\partial}$ is a new screen generated by $F^{w}(\widetilde{J})$ in $\langle b\rangle$ and $\partial$ is an edge on $F^{w}(\widetilde{J})$, and $\Delta_{1}(w b, \widetilde{J}\rangle$ is the union of $\langle w b, \tilde{J}\rangle^{\partial} \backslash\left(\Delta_{0}\langle w b, \tilde{J}\rangle \cup \Delta_{2}\langle w b, \tilde{J}\rangle\right)$ such that $\langle w b, \tilde{J}\rangle^{\partial}$ is a new screen generated by $F^{w}(\widetilde{J})$ in $\langle b\rangle$ and $\partial$ is a vertex on $F^{w}(\tilde{J})$. This definition is the same as we stated in Notation 1 for the case $w=a$ (cf. also Figure 5 again). We also put $\Delta_{0}\langle a, \tilde{J}\rangle=\tilde{J}$.

Assume that $\tilde{J}$ crosses $w b$. We say a screen $K^{\partial} \in N e w\left\langle b c, L^{\partial^{\prime}}\right\rangle$ generated by $F^{b}\left(L^{\partial^{\prime}}\right)$ with $L^{\partial^{\prime}} \in N e w\langle w b, \tilde{J}\rangle$ for some $c \in \mathscr{A}$ is

1. of type 0 , if $\partial \subset F^{b}\left(\Delta_{0}\langle w b, \tilde{J}\rangle\right)^{c l}$,

2. of type 1 , if $\partial \subset F^{b}\left(\Delta_{1}\langle w b, \tilde{J}\rangle\right)$ and it is not of type 0 ,

3. of type 2 , otherwise.

DEFINITION. We denote by $F \tilde{\mathscr{A}}$ the set of new screens generated by $F^{a}\left(a^{\partial}\right)$ in some $\langle b\rangle\left(a, b \in \mathscr{A}, \partial \in D^{\langle a\rangle}\right)$, that is, $F \tilde{A}=\bigcup_{a, b \in \mathscr{A}} \bigcup_{\partial \in D^{\langle a\rangle}} N e w\left\langle a b, a^{\partial}\right\rangle$. For $n \geq 2$, let

$$
F^{n} \tilde{\mathscr{A}}=\bigcup_{|w|=n} \bigcup_{b \in \mathscr{A}} \bigcup_{\partial \in D^{\lfloor w[1]\rangle}} N e w\left\langle w b, w[1]^{\partial}\right\rangle \backslash\left(\bigcup_{k=1}^{n-1} F^{k} \tilde{\mathscr{A}}\right) .
$$

Note that the screen $\tilde{K}$ which is of type 1 belongs to $\bigcup_{k \geq 1} F^{k} \tilde{\mathscr{A}}$.

REMARK 6. (1) $F^{n} \tilde{\mathscr{A}}$ is not the set $\left\{F^{n}(\tilde{a}): \tilde{a} \in \mathscr{A}\right\}$.

(2) In Figure 7, (1) is mapped to (1) and (3) by $F^{a}$, (2) is mapped to (2) by $F^{a}$, 

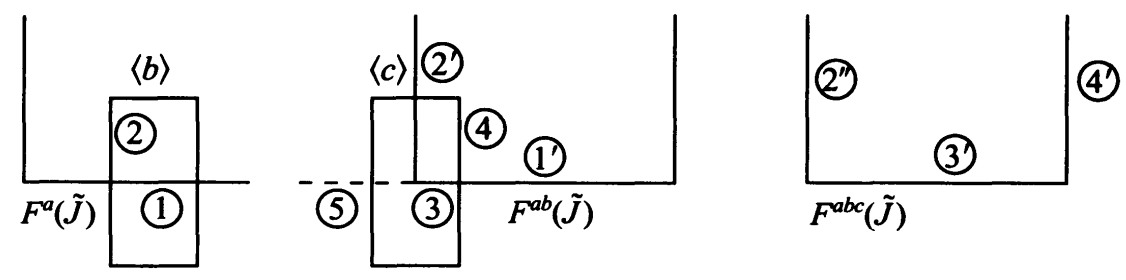

Figure 7

and it is mapped to (2) by $F^{b}$, and (3) (4) is mapped to (3) (4) by $F^{b}$, respectively. type 0 .

1. If a face belongs to (1), (3) or (3), a screen determined by the face is of

2. If a face belongs to (2), (2) or (4), a screen determined by the face is of type 1 .

3. If a face belongs to (1) or (5), a screen determined by the face is of type 2 .

4. If a face belongs to (2) or (4), a screen determined by the face belongs to $F \tilde{A}$.

5. If a face belongs to (2), a screen determined by the face belongs to $F^{2} \mathscr{A}$.

Now we will explain the steps of constructing renewal equations.

1. Construct a renewal equation on $\bigcup_{n=0}^{\infty} F^{n} \mathscr{A}$. In this case, the corresponding Fredholm matrix becomes an infinite dimensional matrix.

2. Construct finite dimensional Fredholm matrices on $\bigcup_{n=0}^{N} F^{n} \tilde{\mathscr{A}}$ renewing repeatedly $\bigcup_{n=N+1}^{\infty} F^{n} \tilde{\mathscr{A}}$ terms appeared in the renewal equation which we got above.

In this section, we will construct a formal renewal equation of the first step, which depends all the elements which belong to $\bigcup_{k=0}^{\infty} F^{k} \tilde{A}$. From this formal equation, we can construct an infinite dimensional Fredholm matrix $\Phi(z)$ and an infinite dimensional vector $\chi_{g}(z)$ such that

$$
s_{g}(z)=\chi_{g}(z)+\Phi(z) s_{g}(z)
$$

where $s_{g}(z)=\left(s_{g}^{\mathcal{J}}(z)\right) \tilde{J}_{\in} \bigcup_{n=0}^{\infty} F^{n} \tilde{\mathscr{A}} \cup I$.

LEMMA 3.3. Let $w \in \mathscr{W}$ be $a$ word and $\tilde{J}$ be a screen of a polygon $J \subset\langle a\rangle(a \in \mathscr{A})$. For a screen $L$ which is generated by some $F(\tilde{K})(\tilde{K} \in N e w\langle w, \tilde{J}\rangle$ in $\langle b\rangle(b \in \mathscr{A}))$, we denote by $G(\tilde{L})$ the set of $\tilde{K} \in N e w\langle w, \tilde{J}\rangle$ such that $F(\tilde{K})$ generates $\tilde{L}$. Then we get

$$
\sum_{\tilde{K} \in G(\tilde{L})} s(\tilde{K})= \begin{cases}+1 / 2 & \text { if } \tilde{L} \text { is of type } 0 \text { or type } 1, \\ 0 & \text { if } \tilde{L} \text { is of type } 2 .\end{cases}
$$

We illustrate the simplest case in Figure 8. Let (1), (2), (3) be the images of $\tilde{K}_{1}$, $\tilde{K}_{2}, \widetilde{K}_{3} \in N e w\langle w, \tilde{J}\rangle$, respectively. Then

1. $\langle b\rangle$ is like (1), then $G(\tilde{L})=\left\{\tilde{K}_{1}, \tilde{K}_{2}, \tilde{K}_{3}\right\}$,

2. $\langle b\rangle$ is like (2), then $G(\tilde{L})=\left\{\tilde{K}_{1}\right\}$,

3. $\langle b\rangle$ is like (3), then $G(\tilde{L})=\left\{\widetilde{K}_{1}, \widetilde{K}_{2}\right\}$.

Proof. As we show in Figure 9, note that 

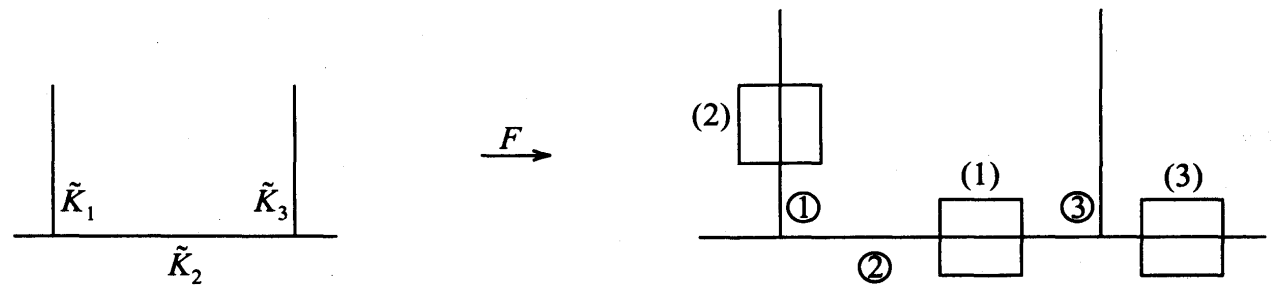

FIGURE 8
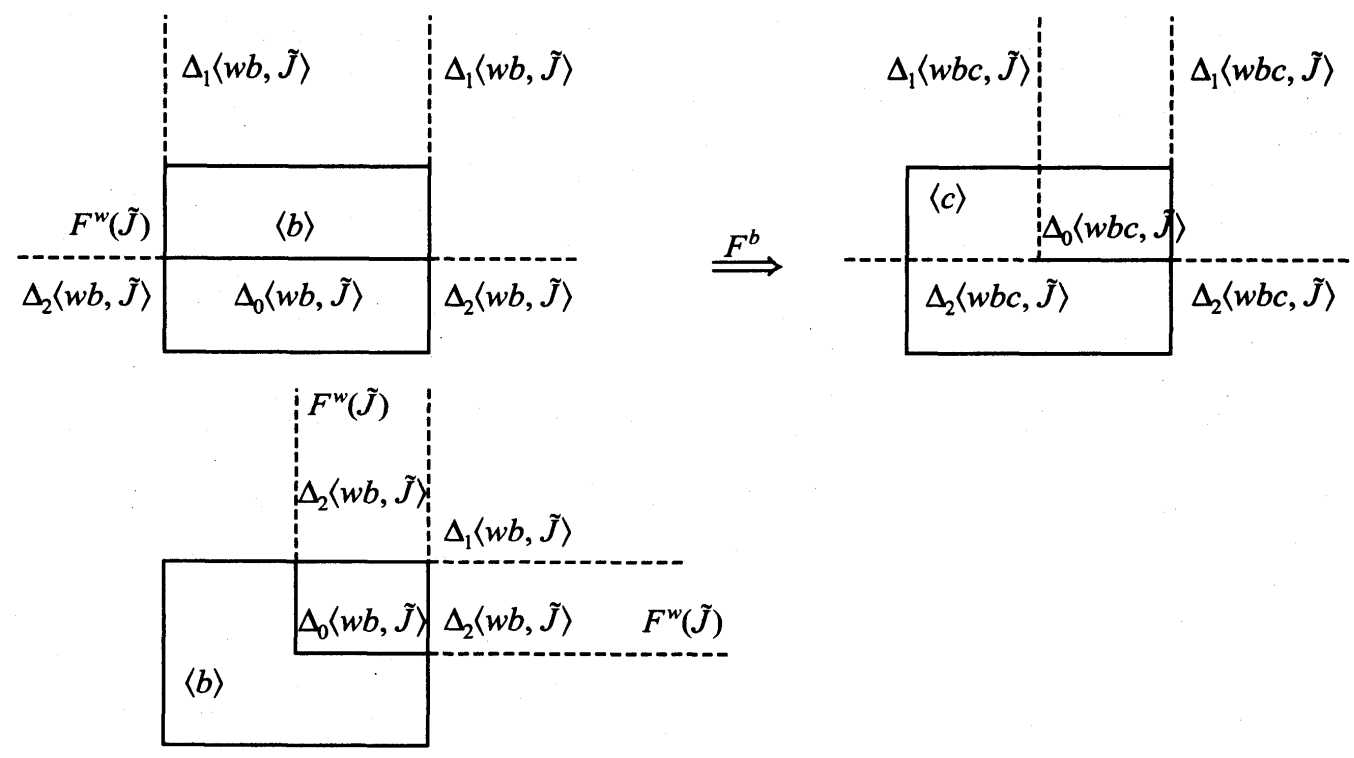

FIGURE 9

1. $\Delta_{0}\langle w b, \tilde{J}\rangle$ is a union of segments (or a segment). Therefore for $\tilde{L}$ of type 0 , $G(\tilde{L})$ is a set of one screen determined by one edge and two screens determined by vertices.

2. $\Delta_{1}\langle w b, \tilde{J}\rangle$ is a union of half lines which is generated by vertices. Therefore for $\tilde{L}$ of type $1, G(\tilde{L})$ is a set of one screen determined by a vertex.

3. $\Delta_{2}\langle w b, \widetilde{J}\rangle$ is a union of half lines and $\Delta_{2}\langle w b, \widetilde{J}\rangle \cup \Delta_{0}\langle w b, \widetilde{J}\rangle$ is a line or a union of half lines. Therefore for $\tilde{L}$ of type $2, G(\tilde{L})$ is a set of one screen determined by an edge and one screen determined by a vertex.

Taking into account the signs of edges and vertices, this completes the proof of Lemma 3.3.

Set for a screen $\widetilde{J}$ of a polygon $J$,

$$
\chi_{g}^{J}(z)= \begin{cases}\tilde{\chi}_{g}^{\mathcal{J}}(z) & \text { if } \tilde{J} \in \bigcup_{k=0}^{\infty} F^{k} \tilde{\mathscr{A}}, \\ \tilde{\chi}_{g}^{J}(z)+\sum_{\substack{w \in \mathcal{W} \\|w| \geq 2}} \sum_{\substack{\tilde{K} \in N e w<w, \tilde{J}\rangle \\ \text { type } 0}} z^{|w|-1} 2 s(\tilde{K}) \eta(w[1,|w|-1]) \tilde{\chi}_{g}^{\tilde{K}}(z) & \text { otherwise, }\end{cases}
$$

and for a screen $\tilde{J} \in \bigcup_{k=0}^{\infty} F^{k} \tilde{\mathscr{A}}(J \subset\langle a\rangle$ for some $a \in \mathscr{A})$ and $\tilde{L} \in \bigcup_{k=0}^{\infty} F^{k} \tilde{\mathscr{A}}$ 


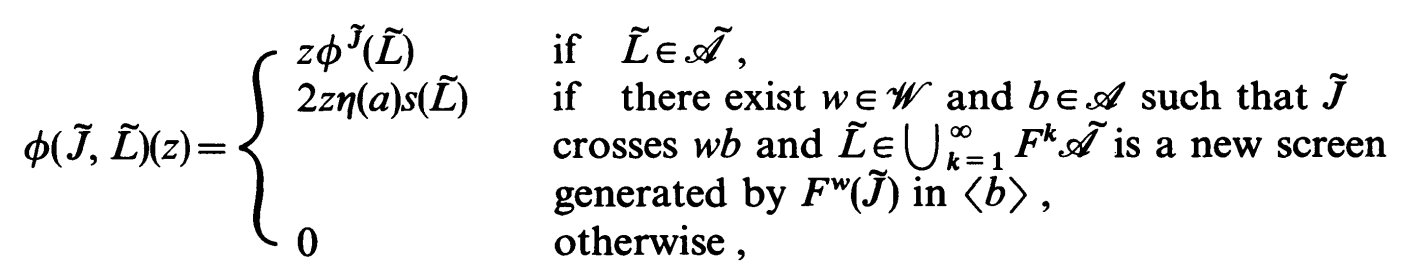

and for a screen $\tilde{J} \notin \bigcup_{k=0}^{\infty} F^{k} \tilde{\mathscr{A}}, J \subset\langle a\rangle$ for some $a \in \mathscr{A}$ and $\tilde{L} \in \bigcup_{k=0}^{\infty} F^{k} \tilde{\mathscr{A}}$

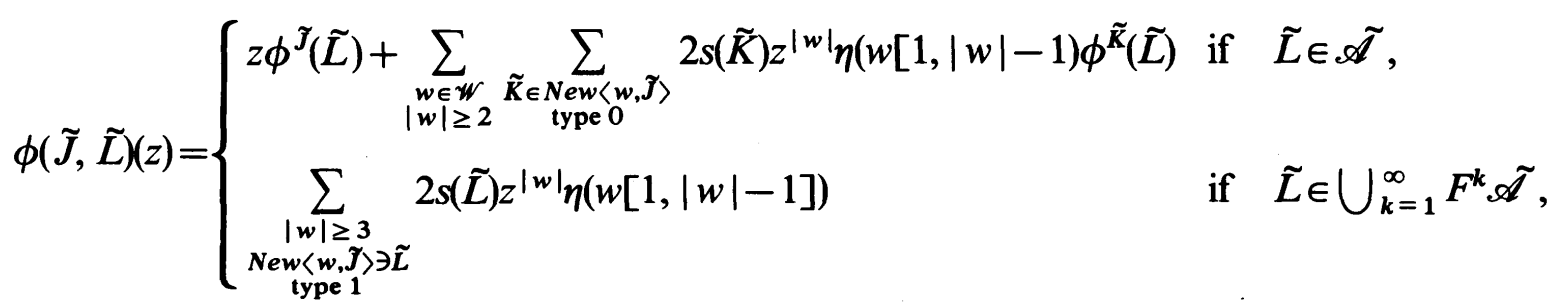

$$
\begin{aligned}
& \phi(\tilde{J}, I)(z)=\left\{\begin{array}{lc}
z^{2} \phi^{J}(I) & \text { if } \tilde{J} \in \bigcup_{k=0}^{\infty} F^{k} \tilde{\mathscr{A}}, \\
z^{2} \phi^{J}(I)+\sum_{\substack{w \in \mathscr{W} \\
|w| \geq 2}} \sum_{\substack{\tilde{K} \in N e w w, \gamma\rangle \\
\text { ype } 0}} 2 s(\tilde{K}) z^{|w|+1} \eta\left(w[1,|w|-1) \phi^{\tilde{K}}(I)\right. & \text { otherwise } .
\end{array}\right.
\end{aligned}
$$

LEMMA 3.4. For all $a \in \mathscr{A}$, polygons $J \subset\langle a\rangle$ and $g \in L^{\infty}$, the following equation (12) formally holds.

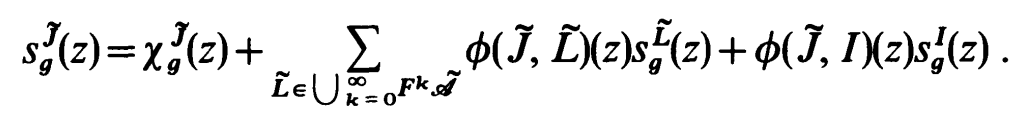

Proof. Recall the second renewal equation (11):

$$
\begin{aligned}
s_{g}^{\mathcal{J}}(z)= & \tilde{\chi}_{g}^{\mathcal{J}}(z)+\sum_{\tilde{b} \in \tilde{\mathscr{A}}} z \phi^{\mathcal{J}}(\tilde{b}) s_{g}^{\tilde{b}}(z)+z^{2} \phi^{\mathcal{J}}(I) s_{g}^{I}(z) \\
& +2 z \eta(a) \sum_{b \in \mathscr{A}} \sum_{\tilde{\mathbf{K}} \in N \in w\langle a b, \tilde{J}\rangle} s(\tilde{K}) s_{g}^{\tilde{\tilde{K}}}(z) .
\end{aligned}
$$

Hence if $\tilde{J} \in \bigcup_{k=0}^{\infty} F^{k} \mathscr{A}$, then $N e w\langle a b, \tilde{J}\rangle \subset \bigcup_{k=1}^{\infty} F^{k} \tilde{\mathscr{A}}$. Therefore it becomes

$$
s_{g}^{J}(z)=\tilde{\chi}_{g}^{J}(z)+\sum_{\tilde{L} \in \bigcup_{k=0}^{\infty} F^{k} \tilde{\mathscr{A}}} \phi(\tilde{J}, \tilde{L})(z) s_{g}^{\tilde{L}}(z)+\phi(\tilde{J}, I) s_{g}^{I}(z) .
$$

This proves (12) for $\tilde{J} \in \bigcup_{k=0}^{\infty} F^{k} \tilde{\mathscr{A}}$. For other cases, we will renew $s_{g}^{\tilde{K}}(z)$ in the last term of (13) repeatedly.

$$
\begin{aligned}
s_{g}^{\gamma}(z)= & \tilde{\chi}_{g}^{\gamma}(z)+\sum_{\tilde{b} \in \tilde{A}} z \phi^{\gamma}(\tilde{b}) s_{g}^{\tilde{b}}(z)+z^{2} \phi^{\gamma}(I) s_{g}^{I}(z) \\
& +\sum_{b \in \mathscr{A}} \sum_{\tilde{K} \in N e w\langle a b, \tilde{\gamma}\rangle} 2 z \eta(a) s(\widetilde{K}) s_{g}^{\tilde{\tilde{K}}}(z)
\end{aligned}
$$




$$
\begin{aligned}
& =\tilde{\chi}_{g}^{\mathcal{J}}(z)+\sum_{\tilde{b} \in \tilde{A}} z \phi^{\tilde{J}}(\tilde{b}) s_{g}^{\tilde{b}}(z)+z^{2} \phi^{\mathcal{J}}(I) s_{g}^{I}(z) \\
& +\sum_{b \in \mathscr{A}} \sum_{\tilde{K} \in N e w\langle a b, J\rangle} 2 z \eta(a) s(\tilde{K})\left[\tilde{\chi}_{g}^{\tilde{K}}(z)+\sum_{\tilde{c} \in \tilde{\mathscr{A}}} z \phi^{\tilde{K}}(\tilde{c}) s_{g}^{\tilde{c}}(z)\right. \\
& \left.+z^{2} \phi^{\tilde{K}}(I) s_{g}^{I}(z)+\sum_{c \in \mathscr{A}} \sum_{\tilde{L} \in N e w\langle b c, \tilde{K}\rangle} 2 z \eta(b) s(\tilde{L}) s_{g}^{\tilde{L}}(z)\right] \\
& =\tilde{\chi}_{g}^{\tilde{J}}(z)+\sum_{b \in \mathscr{A}} \sum_{\tilde{K} \in N e w\langle a b, \tilde{J}\rangle} 2 z \eta(a) s(\tilde{K}) \tilde{\chi}_{g}^{\tilde{K}}(z) \\
& +\sum_{\tilde{c} \in \tilde{A}}\left[z \phi^{\mathcal{J}}(\tilde{c})+\sum_{b \in \mathscr{A}} \sum_{\tilde{K} \in N e w\left\langle a b, \gamma^{\prime}\right\rangle} 2 z^{2} \eta(a) s(\tilde{K}) \phi^{\tilde{K}}(\tilde{c})\right] s_{g}^{\tilde{c}}(z) \\
& +\left[z^{2} \phi^{\mathcal{J}}(I)+\sum_{b \in \mathscr{A}} \sum_{\tilde{K} \in N e w\langle a b, \tilde{J}\rangle} 2 z^{3} \eta(a) s(\tilde{K}) \phi^{\tilde{K}}(I)\right] s_{g}^{I}(z) \\
& +\sum_{b \in \mathscr{A}} \sum_{\tilde{K} \in N e w\langle a b, \tilde{J}\rangle} \sum_{c \in \mathscr{A}} \sum_{\tilde{L} \in N e w\langle b c, \tilde{K}\rangle} 4 s(\tilde{K}) s(\tilde{L}) z^{2} \eta(a b) s_{g}^{\tilde{L}}(z) .
\end{aligned}
$$

Hence, by Lemma 3.3, we can divide the last sum of right hand term of (14) into the sums over screens of type 0 and type 1 . Note that a screen $\tilde{L} \in N e w\langle b c, \tilde{K}\rangle$ for $\tilde{K} \in N e w\langle a b, \tilde{J}\rangle$ which does not belong to $N e w\langle a b c, \tilde{J}\rangle$ is of type 2 (cf. Figure 9 again). Hence

$$
\begin{aligned}
& \sum_{b \in \mathscr{A}} \sum_{\tilde{K} \in N e w\langle a b, \tilde{J}\rangle} \sum_{c \in \mathscr{A}} \sum_{\tilde{L} \in N e w\langle b c, \tilde{K}\rangle} 4 s(\tilde{K}) s(\tilde{L}) z^{2} \eta(a b) s_{g}^{\tilde{L}}(z)
\end{aligned}
$$

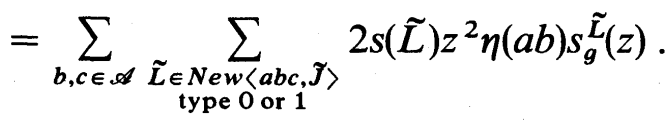

Therefore we get

$$
\begin{aligned}
& \text { rhs. of }(14)=\tilde{\chi}_{g}^{\mathcal{J}}(z)+\sum_{b \in \mathscr{A}} \sum_{\tilde{K} \in N e w\langle a b, J\}} 2 z \eta(a) s(\tilde{K}) \tilde{\chi}_{g}^{\tilde{K}}(z) \\
& +\sum_{\tilde{c} \in \tilde{A}}\left[z \phi^{\tilde{J}}(\tilde{c})+\sum_{b \in \mathscr{A}} \sum_{\tilde{K} \in N e w\langle a b, \tilde{J}\rangle} 2 z^{2} \eta(a) s(\tilde{K}) \phi^{\tilde{K}}(\tilde{c})\right] s_{g}^{\tilde{c}}(z) \\
& +\left[z^{2} \phi^{\mathcal{J}}(I)+\sum_{b \in \mathscr{A}} \sum_{\tilde{K} \in N e w\langle a b, \tilde{J}\rangle} 2 z^{3} \eta(a) s(\tilde{K}) \phi^{\tilde{K}}(I)\right] s_{g}^{I}(z)
\end{aligned}
$$

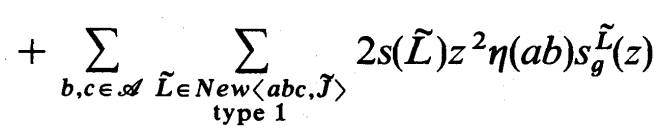

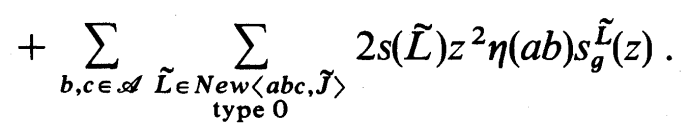


As we remarked before, $\tilde{K}$ of type 1 belongs to $\bigcup_{k=1}^{\infty} F^{k} \tilde{\mathscr{A}}$. Therefore we need only renew the type 0 screens. Repeating this procedure, we get as a formal power series:

$$
\begin{aligned}
& \text { rhs. of }(15)=\tilde{\chi}_{g}^{\mathcal{J}}(z)+\sum_{b \in \mathscr{A}} \sum_{\tilde{K} \in N e w\langle a b, J\rangle} 2 z \eta(a) s(\tilde{K}) \tilde{\chi}_{g}^{\tilde{K}}(z)
\end{aligned}
$$

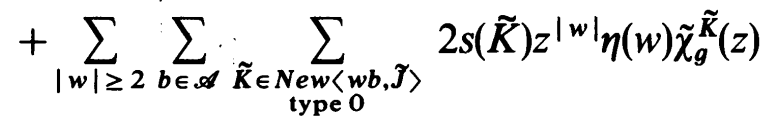

$$
\begin{aligned}
& +\sum_{\tilde{\tilde{c}} \in \tilde{A}}\left[z \phi^{\mathcal{J}}(\tilde{c})+\sum_{b \in \mathscr{A}} \sum_{\tilde{K} \in N e w\langle a b, \tilde{J}\rangle} 2 s(\tilde{K}) z^{2} \eta(a) \phi^{\tilde{K}}(\tilde{c})\right. \\
& +\left(z^{2} \phi^{\mathcal{J}}(I)+\sum_{|w| \geq 2} \sum_{\tilde{K} \in N e w\langle w, J\rangle} z^{|w|+1} 2 s(\tilde{K}) \eta\left(w[1,|w|-1) \phi^{\tilde{K}}(I)\right) s_{g}^{I}(z)\right.
\end{aligned}
$$

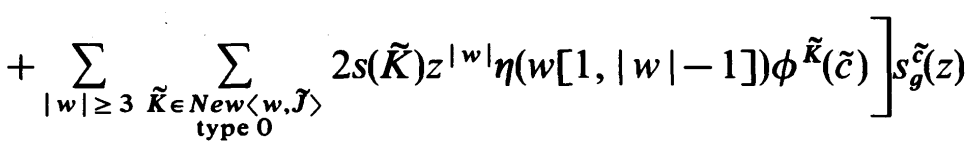

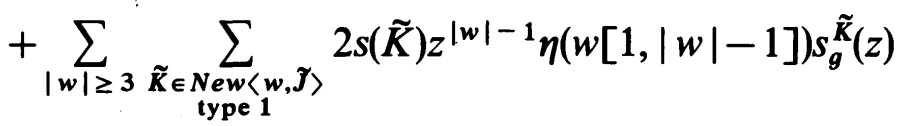

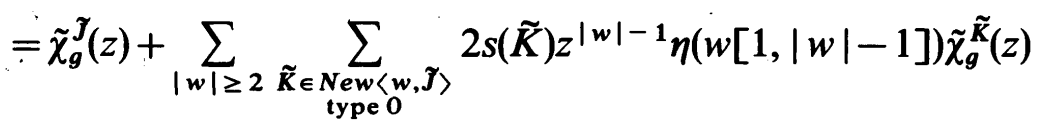

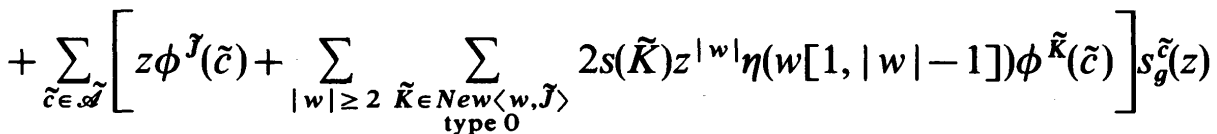

$$
\begin{aligned}
& +\left[z^{2} \phi^{\mathcal{J}}(I)+\sum_{|w| \geq 2} \sum_{\tilde{K} \in N e w\langle w, J\rangle} z^{|w|+1} 2 s(\tilde{K}) \eta(w[1,|w|-1]) \phi^{\tilde{K}}(I)\right] s_{g}^{I}(z)
\end{aligned}
$$

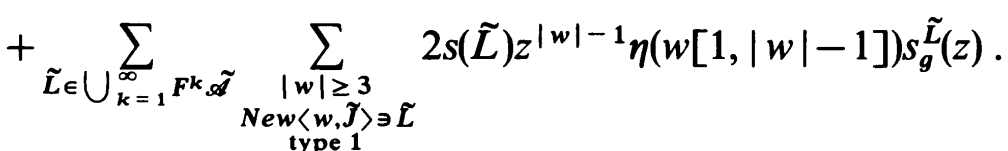

This proves Lemma 3.3.

Using (12) for $\tilde{a} \in \tilde{\mathscr{A}}$ and (9), we can construct a formal renewal equation of the form

$$
s_{g}(z)=\chi_{g}(z)+\Phi(z) s_{g}(z),
$$

where $s_{g}(z), \chi_{g}(z)$ are $\bigcup_{n=0}^{\infty} F^{n} \tilde{\mathscr{A}} \cup I$ vector and $\Phi(z)$ is $\bigcup_{n=0}^{\infty} F^{n} \tilde{\mathscr{A}} \cup I$ matrix and

$$
\begin{gathered}
s_{g}(z)_{\tilde{J}}= \begin{cases}s_{g}^{\mathcal{J}}(z) & \text { if } \tilde{J} \in \bigcup_{n=0}^{\infty} F^{n} \tilde{A}, \\
s_{g}^{I}(z) & \text { if } \quad \tilde{J}=I,\end{cases} \\
\chi_{g}(z)_{\tilde{J}}= \begin{cases}\chi_{g}^{\mathcal{J}}(z) & \text { if } \tilde{J} \in \bigcup_{n=0}^{\infty} F^{n} \tilde{A}, \\
s_{2} \# \mathscr{A} \int g d x & \text { if } \tilde{J}=I,\end{cases}
\end{gathered}
$$




$$
\Phi(z)_{\tilde{J}, \tilde{K}}= \begin{cases}\phi(\tilde{J}, \tilde{K})(z) & \text { if } \tilde{J} \in \bigcup_{n=0}^{\infty} F^{n} \tilde{\mathscr{A}} \\ s(\tilde{K}) & \text { if } \tilde{J}=I \text { and } \tilde{K} \in \tilde{\mathscr{A}} \\ s_{2} z \sum_{a \in \mathscr{A}} \eta(a) & \text { if } \tilde{J}=\tilde{K}=I \\ 0 & \text { otherwise }\end{cases}
$$

\section{Reduction to finite dimension.}

In $\S 3$, we constructed a formal renewal equation which leads to an infinite dimensional Fredholm matrix $\Phi(z)$. Now in this section, we will reduce it to finite dimensional matrices $\Phi_{n}(z)$ for $n=0,1, \cdots$.

The idea is only to renew all the terms $s_{g}^{\tilde{L}}(z)\left(\tilde{L} \in \bigcup_{k=n+1}^{\infty} F^{k} \mathscr{A}\right)$ in the right hand term in (12) repeatedly. Then we will get a renewal equation with only the elements $s_{g}^{\tilde{L}}(z)\left(\tilde{L} \in \bigcup_{k=0}^{n} F^{k} \tilde{A}\right)$. Since screens of type 0 and 1 generate new branches, we need to estimate their numbers. We will prepare several notations.

Set $B_{k}\langle w b, \widetilde{J}\rangle$ be the set of $K^{\partial} \in F^{k} \tilde{\mathscr{A}}$ such that $\tilde{J}$ crosses $w$, and $K^{\partial}$ is of type 1 generated by some $F^{w[|w|]}(\tilde{L})(\tilde{L} \in N e w\langle w, \tilde{J}\rangle)$, that is, the face which corresponds to $\tilde{L}$ lies on $F^{w[|w|-k,|w|] b} \Delta_{1}(w[1,|w|-k], \widetilde{J})$. Note that if $|w| \leq k$, then $B_{k}\langle w b, \widetilde{J}\rangle=\varnothing$, and $B_{0}\langle w b, \widetilde{J}\rangle=\varnothing$ for any $w \in \mathscr{W}$ and $b \in \mathscr{A}$. Let

$$
C_{n, 1}\langle w b, \widetilde{J}\rangle=\bigcup_{k=1}^{n} B_{k}\langle w b, \widetilde{J}\rangle, \quad D_{n, 1}\langle w b, \widetilde{J}\rangle=\bigcup_{k>n} B_{k}\langle w b, \tilde{J}\rangle .
$$

Then using these notations, (12) becomes:

$$
\begin{aligned}
& s_{g}^{\tilde{J}}(z)=\chi_{g}^{\tilde{J}}(z)+\sum_{\tilde{L} \in \bigcup_{k=0}^{\infty} F^{k} \tilde{\mathscr{A}}} \phi(\tilde{J}, \tilde{L})(z) s_{g}^{\tilde{L}}(z)+\phi(\tilde{J}, I)(z) s_{g}^{I}(z) \\
& =\chi_{g}^{\tilde{J}}(z)+\sum_{\tilde{b} \in \tilde{A}} z \tilde{\phi}(\tilde{J}, \tilde{b}) s_{g}^{\tilde{b}}(z)+\phi(\tilde{J}, I)(z) s_{g}^{I}(z) \\
& +\sum_{w \in \mathscr{W},|w| \geq 3} \sum_{\tilde{L} \in \bigcup_{k=0}^{\infty} B_{k}\langle w, \tilde{J}\rangle} 2 s(\tilde{L}) z^{|w|} \eta\left(w[1,|w|-1) s_{g}^{\tilde{L}}(z)\right. \\
& =\chi_{g}^{\tilde{J}}(z)+\sum_{\tilde{b} \in \tilde{A}} z \tilde{\phi}(\tilde{J}, \tilde{b}) s_{g}^{\tilde{b}}(z)+\phi(\tilde{J}, I)(z) s_{g}^{I}(z) \\
& +\sum_{w \in \mathscr{W},|w| \geq 3} \sum_{\tilde{L} \in C_{n, 1}\langle w, J\rangle} 2 s(\tilde{L}) z^{|w|} \eta\left(w[1,|w|-1) s_{g}^{\tilde{L}}(z)\right. \\
& +\sum_{w \in \mathscr{W},|w| \geq 3} \sum_{\tilde{L} \in D_{n, 1}\langle w, J} 2 s(\tilde{L}) z^{|w|} \eta(w[1,|w|-1]) s_{g}^{\tilde{L}}(z) .
\end{aligned}
$$

When we renew $D_{n, 1}\langle w b, \tilde{J}\rangle$, from these screens there appear the screens in $\bigcup_{k=1}^{\infty} F^{k} \mathscr{A}$, which we will denote by $C_{n, 2}\langle w b, \tilde{J}\rangle$ and $D_{n, 2}\langle w b, \tilde{J}\rangle$ contained in $\bigcup_{k=1}^{n} F^{k} \mathscr{A}$ and $\bigcup_{k>n} F^{k} \mathscr{A}$, respectively. Then we again renew $D_{n, 2}\langle w b, \tilde{J}\rangle$, there appear $C_{n, 3}\langle w b, \tilde{J}\rangle$ and $D_{n, 3}\langle w b, \tilde{J}\rangle$ contained in $\bigcup_{k=1}^{n} F^{k} \mathscr{A}$ and $\bigcup_{k>n} F^{k} \tilde{\mathscr{A}}$ respectively, and so on. More 
precisely, for $l \geq 1$ set

$$
\begin{aligned}
& C_{n, l+1}\langle w b, \tilde{J}\rangle=\bigcup_{\substack{J^{\prime} \in D_{n, 1}\left\langle w[1, m], J^{m}\right\rangle \\
m \geq 2}} C_{n, l}\left\langle w[m,|w|] b, \tilde{J}^{\prime}\right\rangle, \\
& D_{n, l+1}\langle w b, \tilde{J}\rangle=\bigcup_{\substack{\left.J^{\prime} \in D_{n, 1}<w[1, m], J^{\prime}\right\rangle \\
m \geq 2}} D_{n, l}\left\langle w[m,|w|] b, \tilde{J}^{\prime}\right\rangle, \\
& C_{n}\langle w b, \tilde{J}\rangle=\bigcup_{l \geq 1} C_{n, l}\langle w b, \tilde{J}\rangle \subset \bigcup_{k=1}^{n} F^{k} \tilde{A} \\
& D_{n}\langle w b, \tilde{J}\rangle=\bigcup_{l \geq 1} D_{n, l}\langle w b, \tilde{J}\rangle \subset \bigcup_{k>n} F^{k} \tilde{\mathscr{A}}
\end{aligned}
$$

REMARK 7. (i) $D_{n}\langle w b, \tilde{J}\rangle=\varnothing$ if $|w|<n$.

(ii) $C_{0}\langle w b, \tilde{J}\rangle=\varnothing$.

(iii) New screens generated by screens in $D_{n}\langle w b, \tilde{J}\rangle$ belong to $\bigcup_{k=1}^{\infty} F^{k} \tilde{\mathscr{A}}$.

Now we will fix $n \geq 0$ and construct the Fredholm matrices which have indices $\bigcup_{k=0}^{n} F^{k} \tilde{\mathscr{A}}$. We need not renew screens which belong to $C_{n}\langle w b, \widetilde{J}\rangle$. Let

$$
N e w_{n}\langle w, \widetilde{J}\rangle=N e w\langle w, \widetilde{J}\rangle \cup D_{n}\langle w, \widetilde{J}\rangle,
$$

and we call a screen of type $(n, 0)$ if it is of type 0 of $N e w_{n}\langle w, \tilde{J}\rangle$ or it belongs to $D_{n}\langle w, \widetilde{J}\rangle$, and of type $(n, 1)$ if it belongs to $C_{n}\langle w, \widetilde{J}\rangle$.

We will explain these notions in Figure 10.

1. Let $F^{a}(\widetilde{J})$ be as in Figure $10(a)$. Screens with faces $\partial\left(\partial \neq F^{b}(A)\right)$ on (2), which is the image of the half line (1) by $F^{b}$, belong to $B_{1}\langle a b \bar{c}, \tilde{J}\rangle$ for $\bar{c} \in \mathscr{A}$ which intersect (2), that is, in Figure 10(b) the screen of the vertex $C$ is an example.

2. Screens with faces $\partial\left(\partial \neq F^{b c}(A)\right)$ on (5), which is the image of (2) by $F^{c}$, belong to $B_{2}\langle a b c \bar{d}, \tilde{J}\rangle$ for $\bar{d} \in \mathscr{A}$ which intersect (5), that is, in Figure 10 (c) the screen of the vertex $E$ is an example.

3. Screens with faces $\partial\left(\partial \neq F^{c}(D)\right)$ on (7), which is the image of (4) by $F^{c}$, belong to $B_{1}\langle a b c \bar{d}\rangle$ for $\bar{d} \in \mathscr{A}$ which intersect (7).

4. To construct $\Phi_{0}(z)$, we will renew the elements of $F \mathscr{A}$. Therefore screens of faces $\partial\left(\partial \neq F^{c}(C)\right)$ on (6), which is the image of (3) by $F^{c}$, belong to $D_{0,2}\left\langle a b c d^{\prime}\right\rangle$, because they belong to $F \tilde{\mathscr{A}}$. The vertices $F, G$ and the edge $F G$ are examples.

5. To construct $\Phi_{1}(z)$, we will renew the elements of $F^{2} \tilde{\mathscr{A}}$. Therefore screens of faces $\partial\left(\partial \neq F^{d}(E)\right)$ on the image of (8) belong to $C_{1,2}\langle a b c d, \widetilde{J}\rangle$, because they belong to $F \tilde{A}$.

6. The Screens of the vertices $A, B$ and the edge $A B$ belong to $N e w\langle a b, \tilde{J}\rangle$.

7. The screens of vertices $C, F^{b}(A), D$ and the edges $C F^{b}(A), F^{b}(A) D$ belong to New $\langle a b c, \widetilde{J}\rangle$.

8. The screens of the vertices $F^{b c}(A), E, H$ and the edge $E F^{b c}(A), F^{b c}(A) H$ belong to $N e w\langle a b c d, \widetilde{J}\rangle$. 


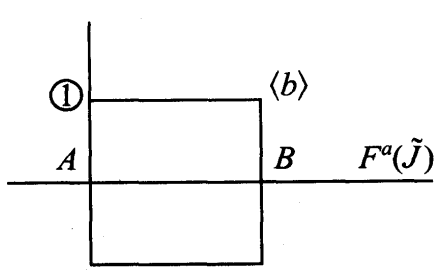

(a)

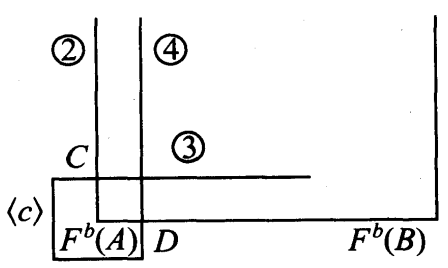

(b)

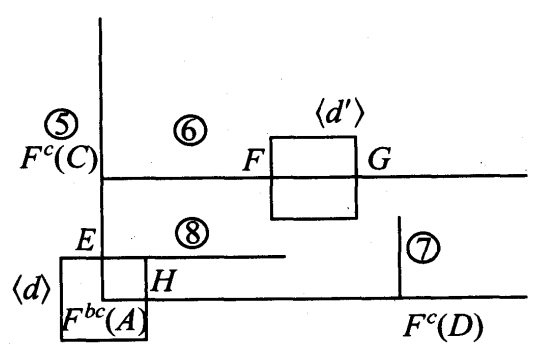

(c)

Figure 10

9. The screens of the vertices $F, G$ and the edge $F G$ belong to $N e w_{0}\left\langle a b c d^{\prime}, \tilde{J}\right\rangle$.

10. New $w_{1}\langle a b c d, \tilde{J}\rangle=N e w\langle a b c d, \tilde{J}\rangle$. There appear screens which belong to $D_{1,2}\langle a b c d e, \tilde{J}\rangle$ for the first time by faces on the image of (8) by $F^{d e}$ for some $e \in \mathscr{A}$ which intersect the image of (8) by $F^{d}$, and $N e w_{1}\langle a b c d e, \tilde{J}\rangle \supsetneqq N e w\langle a b c d e, \tilde{J}\rangle$.

Set for a screen $\tilde{J}$ of a polygon $J$,

$$
\chi_{n, g}^{\tilde{J}}(z)= \begin{cases}\tilde{\chi}_{g}^{\tilde{J}}(z) & \text { if } \tilde{J} \in \bigcup_{k=0}^{n-1} F^{k} \tilde{\mathscr{A}} \cup I, \\ \tilde{\chi}_{g}^{\tilde{J}}(z)+\sum_{\substack{w \in \mathscr{W} \\|w| \geq 2}} \sum_{\substack{\tilde{K} \in N e w_{n}\langle w, \tilde{J}\rangle \\ \operatorname{type}(n, 0)}} 2 s(\tilde{K}) z^{|w|-1} \eta\left(w[1,|w|-1) \tilde{\chi}_{g}^{\tilde{K}}(z)\right. & \text { otherwise, }\end{cases}
$$

and for a screen $\tilde{J} \in \bigcup_{k=0}^{n-1} F^{k} \tilde{\mathscr{A}}(J \subset\langle a\rangle$ for some $a \in \mathscr{A})$

$$
\phi_{n}(\tilde{J}, \tilde{L})(z)= \begin{cases}z \phi^{\tilde{J}}(\tilde{L}) & \text { if } \tilde{L} \in \tilde{\mathscr{A}}, \\ 2 z s(\tilde{J}) \eta(a) & \text { if } \tilde{L} \in \bigcup_{k=1}^{n} F^{k} \tilde{\mathscr{A}} \text { is a new screen generated by } F^{a} \tilde{J}, \\ 0 & \text { otherwise, }\end{cases}
$$

and for a screen $\tilde{J} \notin \bigcup_{k=0}^{n-1} F^{k} \mathscr{A}$

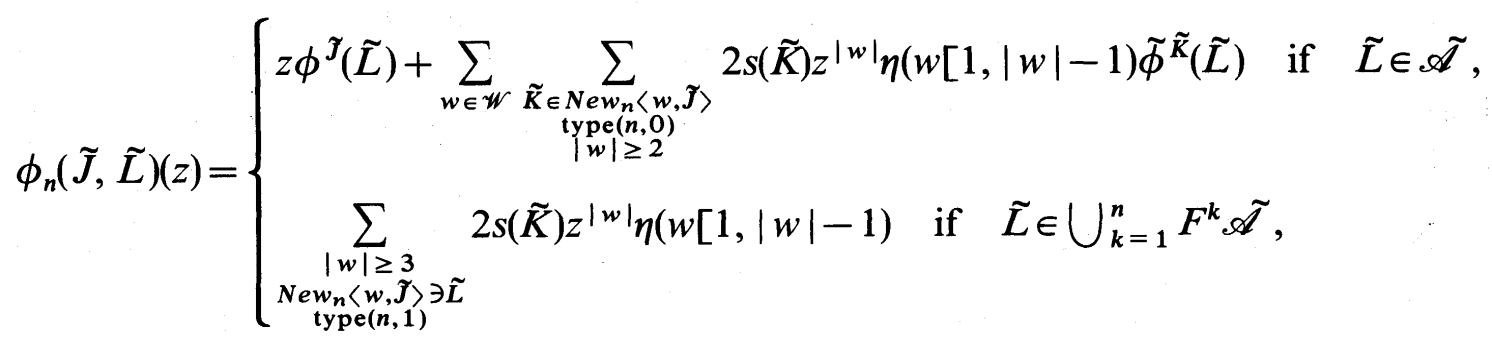




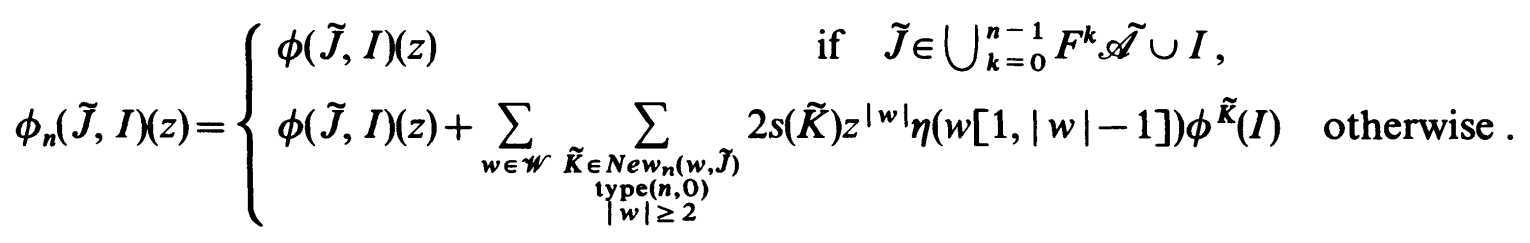

We will discuss the radii of convergences in $\$ 5$.

LEMMA 4.1. For a screen $\widetilde{J}$ of a polygon $J$ such that $J \subset\langle a\rangle(a \in \mathscr{A})$ and $g \in L^{\infty}$, we get

$$
s_{g}^{J}(z)=\chi_{n, g}^{J}(z)+\sum_{\tilde{L} \in \bigcup_{k=0}^{n} F^{k} \tilde{A} \cup I} \phi_{n}(\tilde{J}, \tilde{L})(z) s_{g}^{\tilde{L}}(z) .
$$

Proof. We now renew the last term of the right hand term of (16) again, then

$$
\begin{aligned}
& \sum_{w \in W,|w| \geq 3} \sum_{\tilde{L} \in D_{n, 1}\langle w, J\rangle} 2 s(\tilde{L}) z^{|w|} \eta(w[1,|w|-1]) s_{g}^{\tilde{L}}(z) \\
& =\sum_{w \in W,|w| \geq 3} \sum_{\tilde{L} \in D_{n, 1}\langle w, J\rangle} 2 s(\tilde{L}) z^{|w|} \eta(w[1,|w|-1]) \\
& \times\left[\chi_{g}^{\tilde{L}}(z)+\sum_{\tilde{b} \in \tilde{A}} z \tilde{\phi}(\tilde{L}, \tilde{b}) s_{g}^{\tilde{b}}(z)+\phi(\tilde{L}, I) s_{g}^{I}(z)\right. \\
& +\sum_{w^{\prime} \in W^{\prime}\left|w^{\prime}\right| \geq 3} \sum_{\tilde{K} \in C_{n, 1}\left\langle w^{\prime}, \tilde{L}\right\rangle} 2 s(\tilde{K}) z^{\left|w^{\prime}\right|} \eta\left(w^{\prime}\left[1,\left|w^{\prime}\right|-1\right) s_{g}^{\tilde{K}}(z)\right. \\
& \left.+\sum_{w^{\prime} \in W^{\prime},\left|w^{\prime}\right| \geq 3} \sum_{\tilde{K} \in D_{n, 1}\left\langle w^{\prime}, \tilde{L}\right\rangle} 2 s(\tilde{K}) z^{\left|w^{\prime}\right|} \eta\left(w^{\prime}\left[1,\left|w^{\prime}\right|-1\right]\right) s_{g}^{\tilde{K}}(z)\right] .
\end{aligned}
$$

By Lemma 3.4, new screens which are not of type $(n, 0)$ or $(n, 1)$ will vanish, and in a similar way to prove Lemma 3.3 we get

$$
\begin{aligned}
\text { rhs. of }(18)= & \sum_{w \in \mathscr{W},|w| \geq 3} \sum_{\tilde{L} \in D_{n, 1}\langle w, J\rangle} 2 s(\tilde{L}) z^{|w|} \eta(w[1,|w|-1]) \\
& \times\left[\chi_{g}^{\tilde{L}}(z)+\sum_{\tilde{b} \in \tilde{L}} z \tilde{\phi}(\tilde{L}, \tilde{b}) s_{g}^{\tilde{b}}(z)+\phi(\tilde{L}, I)(z) s_{g}^{I}(z)\right] \\
& +\sum_{w \in W,|w| \geq 6} \sum_{\tilde{L} \in C_{n, 2\langle w, J\rangle}} 2 s(\tilde{L}) z^{|w|} \eta\left(w[1,|w|-1) s_{g}^{\tilde{L}}(z)\right. \\
& +\sum_{w \in W,|w| \geq 6} \sum_{\tilde{L} \in D_{n, 2\langle w, \tilde{L}\rangle}} 2 s(\tilde{L}) z^{|w|} \eta(w[1,|w|-1]) s_{g}^{\tilde{L}}(z) .
\end{aligned}
$$

Therefore we can prove this lemma by renewing repeatedly all the terms which belong to $\bigcup_{w \in W} D_{n}\langle w, \tilde{J}\rangle$.

Now we get a formal renewal equation. 
THEOREM 4.1. Fix any $n \geq 0$. For $g \in L^{\infty}$, we get

$$
\left(I-\Phi_{n}(z)\right) s_{n, g}(z)=\chi_{n, g}(z),
$$

where

$$
\begin{aligned}
& s_{n, g}(z)=\left(s_{g}^{\tilde{K}}(z)\right)_{\tilde{K} \in \bigcup_{k=0}^{n} F^{k} \tilde{\mathscr{A}} \cup I}, \\
& \chi_{n, g}(z)=\left(\chi_{n, g}^{\tilde{K}}(z)\right)_{\tilde{K} \in \bigcup_{k=0}^{n} F^{k} \tilde{A} \cup I}, \\
& \Phi_{n}(z)=\left(\phi_{n}(\tilde{K}, \tilde{L})\right)_{\tilde{K}, \tilde{L} \in \bigcup_{k=0}^{n} F^{k} \tilde{\mathscr{A}} \cup I} .
\end{aligned}
$$

Therefore, in the domain where the coefficients of $\Phi_{n}(z)$ and $\chi_{n, g}(z)$ are analytic, the singularities of $s_{g}^{\tilde{K}}(z)\left(\tilde{K} \in \bigcup_{k=0}^{n} F^{k} \mathscr{A}\right)$ are determined by $\operatorname{det}\left(I-\Phi_{n}(z)\right)=0$.

In $\S 5$, we will show the zeros of the Fredholm determinant $\operatorname{det}\left(I-\Phi_{n}(z)\right)$ correspond to the reciprocals of the eigenvalues of the Perron-Frobenius operator $P$ restricted to a family of functions $\mathscr{B}$.

\section{Spectrum of Perron-Frobenius operator.}

5.1. Estimate of radii of convergences. We will discuss first the radii of convergences of the renewal equations constructed in $\S 4$. Afterwards, we will show an example for which the renewal equations has sufficiently large radii of convergences.

Recall

$$
\xi=\liminf _{n \rightarrow \infty} \underset{x \in I}{\operatorname{essinf}} \frac{1}{n} \log \left|\operatorname{det} D\left(F^{n}\right)(x)\right|
$$

and we define

$$
v=\limsup _{n \rightarrow \infty} \sup _{l} \frac{1}{n} \log \#\{w:|w|=n,\langle w\rangle \cap l \neq \varnothing\},
$$

where $D\left(F^{n}\right)$ is the jacobian matrix of $F^{n}$, and $\sup _{l}$ is the supremum over all segments $l$. We call that $F$ is expanding, if

$$
\underline{\xi}=\liminf _{n \rightarrow \infty} \frac{1}{n} \text { essinf } \log \left[\text { minimum of } \mid \text { the eigenvalue of } D\left(F^{n}\right)(x) \mid\right]>0 .
$$

As we assumed that $F$ is expanding, $F$ is expanding for all the directions. Therefore the set of polygons $\{\langle a\rangle: a \in \mathscr{A}\}$ is a generator, that is, $\left\{F^{k}\langle a\rangle: a \in \mathscr{A}, k=0,1,2, \cdots\right\}$ generates the $\sigma$-algebra.

Lemma 5.1. Assume that $F$ is expanding and $\xi>v$. Then, there exist $\delta_{n}>0$ such that $\delta_{n}$ tend to 0 as $n \rightarrow \infty$, and for any $\varepsilon>0$, in the domain $|z|<e^{\xi-v-\delta_{n}-\varepsilon}, \phi_{n}(\tilde{J}, \tilde{L})(z)$ and $\chi_{n, g}^{\tilde{J}}(z) /\|g\|_{\infty}$ are uniformly bounded in screens $\tilde{J}$ of a polygon $J \subset\langle a\rangle(a \in \mathscr{A})$ and $\tilde{L} \in \bigcup_{k=0}^{n} \tilde{\mathscr{A}}$, where $g \in L^{\infty}$ and $\|\cdot\|_{\infty}$ is the $L^{\infty}$ norm. 
Proof. For any $\varepsilon>0$, the number of words with length $m$ which intersect $\tilde{J}$ is at most $C e^{(v+\varepsilon / 2) m}$ with some constant $C>0$ which is independent of screen $\widetilde{J}$. First note that the number of $\bigcup_{|w|=m} N e w\langle w, \tilde{J}\rangle$ is at most $5 C e^{(v+\varepsilon / 2) m}$, because one screen can generate at most 5 new screens ( 3 vertices and 2 edges) in some $\langle b\rangle(b \in \mathscr{A})$. For a screen $\tilde{L} \in F^{k} \tilde{A}(k \geq 1)$ of type 1 for which $\phi_{n}(\tilde{J}, \tilde{L})(z) \neq 0$, there exists a word $w$ $(|w| \geq 2)$ such that the face which corresponds to $\tilde{L}$ lies on $F^{k}\left(\Delta_{1}\langle w, \tilde{J}\rangle\right)$. Therefore $\tilde{L}$ is generated by $F^{a_{k}^{x}(\tilde{K})}$ where $x \in I$ is one of two endpoints of $\Delta_{0}\langle w, \tilde{J}\rangle, a_{1}^{x} a_{2}^{x} \cdots$ is its expansion, and the face which corresponds to $\tilde{K}$ is a vertex $F^{k}(x)$, that is, $\tilde{K} \in N e w\left\langle w a_{1}^{x} \cdots a_{k}^{x}, \tilde{J}\right\rangle$. Therefore the number of screens belonging to $F^{k}(\tilde{\mathscr{A}})$ of type 1 $(k \geq 1)$ generated by $F(\tilde{K})$ for $\tilde{K} \in \bigcup_{|w|=m-1} N e w\langle w, \widetilde{J}\rangle$ in some $b \in \mathscr{A}$ is at most $C_{0} e^{(v+\varepsilon / 2)(m-k)}$ if $m \geq k \geq 1$ for some constant $C_{0}$, and it equals 0 if $k>m$.

Set

$$
f(m)=\sup _{\tilde{J}} \sum_{|w|=m} N e w_{n}\langle w, \tilde{J}\rangle,
$$

where sup is taken over all screens generated by faces of convex polygons in $I$. Then $f(m)$ equals the sum of the number of $\sup _{|w|=m} N e w\langle w, \tilde{J}\rangle$ and the sum of the number of screens generated by $F^{n}\left(\tilde{J}^{\prime}\right)\left(\tilde{J}^{\prime} \in \bigcup_{|w|=m-n-k} N e w\langle w, \tilde{J}\rangle\right)$ over $k=1$ to $m-n$. Therefore summing all these numbers, we get

$$
f(m) \leq 5 C e^{(v+\varepsilon / 2) m}+C \sum_{k=1}^{m-n} e^{(v+\varepsilon / 2)(m-n-k)} f(k)
$$

for $m \geq n$. Then multiplying both sides by $t^{m}$, taking the sum over $m=1$ to $\infty$, and with a reduction, we have

$$
\sum_{k=1}^{\infty} f(k) t^{k} \leq \frac{5 C e^{v+\varepsilon / 2} t}{1-e^{v+\varepsilon / 2} t-C_{0} e^{v+\varepsilon / 2} t^{n}},
$$

if the denominator is positive. Since the denominator is positive for sufficiently small $t$, taking $\delta_{n}>0$ such that $e^{-v-\varepsilon / 2-\delta_{n}}$ is the minimal root of $1-e^{v+\varepsilon / 2} t-C_{0} e^{v+\varepsilon / 2} t^{n}=0$, we get

$$
f(m) \leq C^{\prime} e^{\left(v+\varepsilon+\delta_{n}\right) m}
$$

with some constant $C^{\prime}$, and $\delta_{n} \rightarrow 0$ as $n \rightarrow \infty$. On the other hand,

$$
\left|\tilde{\chi}_{g}^{J}\right| \leq \operatorname{Lebes}(I)\left(1+|z| \sum_{a \in \mathscr{A}} \eta(a)\right)\|g\|_{\infty},
$$

where Lebes $(J)$ is the Lebesgue measure of a set $J$. Using (19) and (20), we find that there exists a constant $C^{\prime \prime}$ such that the $m$-th coefficient of $\chi_{g}^{J}(z)$ satisfies

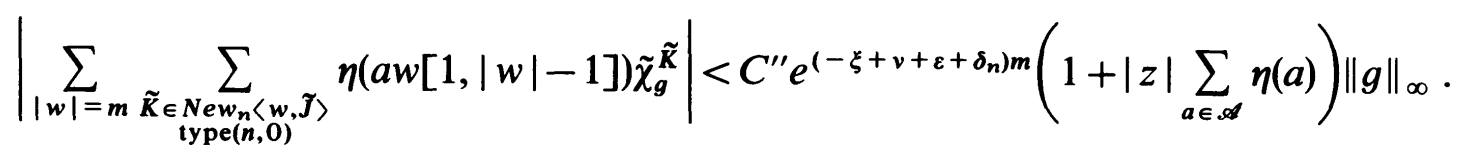


This proves the uniform boundedness of $\chi_{n, g}^{\widetilde{J}} /\|g\|_{\infty}$. To estimate $\phi_{n}$, we only need to estimate the radius of convergence of $\phi_{n}(\tilde{J}, \tilde{K})$ for $\tilde{J} \in F^{n} \tilde{\mathscr{A}}$ and $\tilde{L} \in \tilde{\mathscr{A}}$. Since $\phi^{\tilde{K}}(\tilde{L})$ is bounded, we also only need to calculate the number of elements $\bigcup_{|w|=m} N e w_{n}\langle w, \tilde{J}\rangle$. Thus there exists some constant $C^{\prime \prime \prime}$ such that

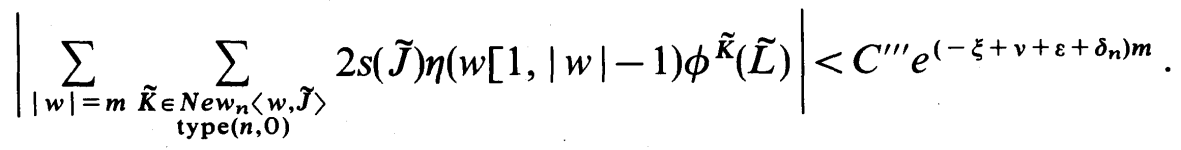

This proves the lemma.

LEMMA 5.2. Assume that $F$ is expanding and $\xi>v$. Then in the domain $|z|<e^{\xi-v-\delta_{n}}$, $\operatorname{det}\left(I-\Phi_{n}(z)\right)$ is analytic.

5.2. An example. Roughly speaking, as $F^{n}$ expands all the directions at most in the order $e^{(\xi-\xi) n}$, the length of an edge of a polygon $\langle w\rangle$ with $|w|=n$ must be longer than $e^{-(\xi-\xi) n}$. Therefore, for any segment of unit length, the number of words with length $n$ which intersect this segment is at most $e^{(\xi-\xi) n}$. Hence $v$ may equal $\xi-\underline{\xi}$. Namely, as shown in the former subsection, the radii of convergences of the Fredholm matrices etc. may be larger than or equal to $e^{\xi-\underline{\xi}}$. This is true for Markov transformations, but for non-Markov transformations, there may appear small polygons $\langle w\rangle$ comparing its length $|w|$. Thus, it may happen $v$ become very large. We will give a non-Markov example for which $\xi>v$.

ExAmple 2. Consider a triangle $A B C$. On the segment $A B$ take points $D, P, Q$. Assume that $A D<A C<A P$ and $B D<B C<B Q$ (cf. Fig. 11). Let $\mathscr{A}=\{a, b\},\langle a\rangle=$ $\triangle A C D$ and $\langle b\rangle=\triangle B C D$. $A$ transformation $F^{a}$ maps $A, C$ and $D$ to $A, P$ and $C$, respectively. $A$ transformation $F^{b}$ maps $B, C$ and $D$ to $B, Q$ and $C$, respectively. Indeed, take

$$
\begin{aligned}
A=\left(\begin{array}{l}
1 \\
1
\end{array}\right), B=\left(\begin{array}{l}
0 \\
0
\end{array}\right), C=\left(\begin{array}{l}
1 \\
0
\end{array}\right), D=\left(\begin{array}{l}
d \\
d
\end{array}\right), P=\left(\begin{array}{l}
p \\
p
\end{array}\right) \text { and } Q=\left(\begin{array}{l}
q \\
q
\end{array}\right) \text {. Then } \\
F^{a}\left(\begin{array}{l}
x \\
y
\end{array}\right)=M^{a}\left[\left(\begin{array}{l}
x \\
y
\end{array}\right)-\left(\begin{array}{l}
1 \\
1
\end{array}\right)\right]+\left(\begin{array}{l}
1 \\
1
\end{array}\right), \quad F^{b}\left(\begin{array}{l}
x \\
y
\end{array}\right)=M^{b}\left(\begin{array}{l}
x \\
y
\end{array}\right), \\
M^{a}=\left(\begin{array}{cc}
p-1 & 1-p \\
1 /(1-d)+p-1 & 1-p
\end{array}\right), \quad M^{b}=\left(\begin{array}{cc}
q & 1 / d-q \\
q & -q
\end{array}\right),
\end{aligned}
$$

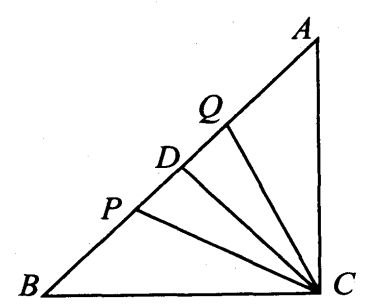

Figure 11 
and the eigenvalues of $M^{a}$ and $M^{b}$ are $\pm \sqrt{(1-p) /(1-d)}$ and $\pm \sqrt{q / d}$, respectively. Therefore, $F$ is expanding for $0<p<d<q$.

LEMMA 5.3. For the mapping $F$ of the example, there exists a constant $C>0$ such that a segment shorter than $C$ can be divided at most 4 segments by 3 iterations of $F$.

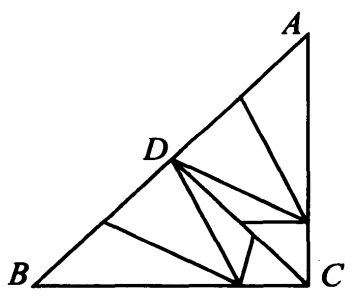

FIGURE 12

ProOF. From Figure 12, at most 4 polygons corresponding to words with length 3 have same vertex. To be divided into more than 4 segments by 3 iterations of $F$, a segment must cross more than 4 polygons corresponding to words with length 3 . Hence it must be longer than some constant $C>0$. This proves the lemma.

LEMMA 5.4. Let $F$ be a mapping with $\mathscr{A}=\{a, b\}$ for which there exists a constant $C>0$ such that a segment shorter than $C$ can be divided at most 4 segments by 3 iterations of $F$. Then the maximal number of words with length $n$ which intersect a screen is at most the order $\max \left\{2^{2 n / 3}, e^{(\xi-\xi) n}\right\}$.

ProOF. Let $a_{n}$ be the number of words with length $n$ which intersect a screen $\tilde{J}$. Then each pieces of $\tilde{J} \cap\langle w\rangle(|w|=n)$ can be divided into three types.

1. $\tilde{J} \cap\langle w\rangle$ is divided into two pieces contained in words $w a$ and $w b(a, b \in \mathscr{A})$, but both of them are contained in some words with length $n+2$, that is, for example, $\tilde{J} \cap\langle w a\rangle \subset\langle w a a\rangle$ or $\widetilde{J} \cap\langle w a\rangle \subset\langle w a b\rangle(\langle w a)=\langle w a a\rangle$ or $\langle w a b\rangle)$. We denote the number of such $\tilde{J} \cap\langle w\rangle$ by $b_{n}$.

2. $\tilde{J} \cap\langle w\rangle$ is divided into two pieces contained in words $w a, w b$, and at least one of them again divided into two pieces contained in words waa, wab, wba or wbb. We denote the number of such $\widetilde{J} \cap\langle w\rangle$ by $c_{n}$.

3. $\tilde{J} \cap\langle w\rangle$ is contained in $\langle w a\rangle$ or $\langle w b\rangle$, that is, $\langle w\rangle=\langle w a\rangle$ or $\langle w b\rangle$. We denote the number of such $\widetilde{J} \cap\langle w\rangle$ by $d_{n}$.

Of course, $a_{n}=b_{n}+c_{n}+d_{n}$. From the notation, we get

$$
a_{n+1}=2 b_{n}+2 c_{n}+d_{n}, \quad a_{n+2} \leq 2 b_{n}+4 c_{n}+2 d_{n} .
$$

Now, some of the segments $\widetilde{J} \cap\langle w\rangle$ corresponding to $c_{n}$ are divided into segments by words with length $n+3$, but the length of such $F^{n}(\widetilde{J} \cap\langle w\rangle)$ must be longer than $C$. On the other hand, the total length of $F^{n}(\widetilde{J})$ is at most constant times $e^{(\xi-\xi) n}$. Therefore, there exists a constant $C^{\prime}>0$ such that 


$$
a_{n+3} \leq 4 b_{n}+4 c_{n}+4 d_{n}+C^{\prime} e^{(\xi-\xi) n}=4 a_{n}+C^{\prime} e^{(\xi-\xi) n} .
$$

Therefore $a_{n}$ is less than or equal to the order

$$
C^{\prime} e^{(\xi-\xi) n}\left(1+2^{2} e^{-(\xi-\xi) 3}+\cdots+\left(2^{2} e^{-(\xi-\xi) 3}\right)^{[n / 3]}\right) .
$$

This shows

1. if $4 e^{-(\xi-\xi) 3}<1$, then $a_{n}$ is of the order $e^{(\xi-\xi) n}$,

2. if $4 e^{-(\xi-\xi) 3} \geq 1$, then $a_{n}$ is of the order $2^{2 n / 3}$.

This proves the lemma.

Summarizing the results, we get:

Proposition 5.1. The above example satisfies $\xi>v$ if $\xi>\frac{2}{3} \log 2$.

REMARK 8. We can prove the following in a same way as above: For a mapping $F$, there exists $n$ such that there exists a constant $C>0$ such that a segment shorter than $C$ can be divided into at most $N$ segments by $n$ iterations of $F$. Then $F$ satisfies $\xi>v$ if $\xi>\frac{1}{n} \log N$.

5.3. Space $\mathscr{B}$. We will study the sapce $\mathscr{B}$ which is an extension of $B V$ in one-dimensional case. When we restrict the domain of the Perron-Frobenius operator $P$ to this $\mathscr{B}$, we can prove the similar results as in one-dimensional cases.

DEFINITION. Let $\mathscr{B}=\mathscr{B}(F)$ be the set of functions $f \in L^{1}$ for which there exists $\left\{C_{w}\right\}_{w \in W}$ such that $f_{m}(x)=\sum_{|w| \leq m} C_{w} 1_{\langle w\rangle}(x)$ converges to $f$ in $L^{1}$ and that

$$
\sum_{n=1}^{\infty} e^{-v^{\prime} n} \sum_{|w|=n}\left|C_{w}\right|<\infty
$$

for any $v^{\prime}>v$.

Set for $f \in \mathscr{B}$ and $v^{\prime}>v$

$$
\|f\|_{v^{\prime}}=\inf \sum_{n=1}^{\infty} e^{-v^{\prime} n} \sum_{|w|=n}\left|c_{w}\right|
$$

where infimum is taken over all $\left\{C_{w}\right\}_{w \in W}$ which satisfy the above condition.

REMARK 9. This is an extension of $B V$ in one-dimensional case, since for $f \in B V$ there exists $\left\{C_{w}\right\}_{w \in W}$ such that $\sum_{n=1}^{\infty} r^{n} \sum_{|w|=n}\left|C_{w}\right|<\infty$ for any $0<r<1$ (cf. [10]). Note that $\mathscr{B}$ in one-dimensional case is a bit wider class than $B V$. Because the definition of

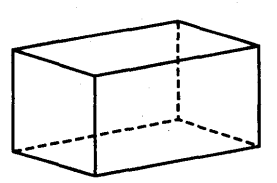

by alphabets

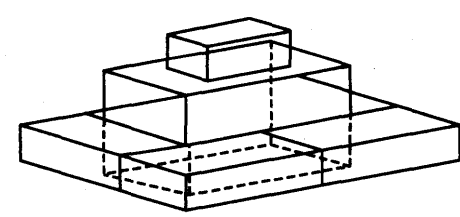

by words $|w| \leq 2$

FIGURE 13. Approximation of a function 
$\mathscr{B}$ depends on a mapping $F$, the above definition may seem to be unnatural. However, the number of words at $n$-th step of an approximation of a mountain-like function by indicator functions of words as in Figure 13 is asymptotically $e^{v n}$, therefore any function which has finite numbers of 'mountains' and 'valleys' belongs to $\mathscr{B}$. Thus the set $\mathscr{B}$ is sufficiently rich.

By the norms $\|\cdot\|_{\nu^{\prime}}, \mathscr{B}$ becomes a locally convex linear space, and $P f \in \mathscr{B}$ holds for $f \in \mathscr{B}$. Put for $f \in L^{1}$ and $g \in L^{\infty}$

$$
(f, g)(z)=\sum_{n=0}^{\infty} z^{n} \int P^{n} f(x) g(x) d x .
$$

Lemma 5.5. Assume that $F$ is expanding and $\xi>v$. Then in the domain $|z|<e^{\xi-v-\delta_{n}}$,

$$
\sup _{\|f\|_{\nu^{\prime}=1}} \sup _{\|g\|_{\infty}=1}|(f, g)(z)|<\infty
$$

for any $v^{\prime}>v$, if and only if $\operatorname{det}\left(I-\Phi_{n}(z)\right) \neq 0$.

Proof. Take any $\varepsilon>0$ and fix it. For simplicity, we only consider $g \in L^{\infty}$ such that $\|g\|_{\infty}=1$. Take $f_{m}=\sum_{|w| \leq m} C_{w} 1_{\langle w\rangle}$ which converges to $f \in \mathscr{B}$ in $L^{1}$, which satisfies $\sum_{|w|=n}\left|C_{w}\right| \leq\|f\|_{v^{\prime}} e^{\left(v^{\prime}+\varepsilon\right) n}$ for sufficiently large $n$. Note that, since $f_{m} \rightarrow f$ in $L^{1}$, in $|z|<1$

$$
\begin{aligned}
& \left|\left(f_{m}, g\right)(z)-(f, g)(z)\right|=\left|\sum_{n=0}^{\infty} z^{n} \int\left(f_{m}(x)-f(x)\right) g\left(F^{n}(x)\right) d x\right| \\
& \left.\leq \sum_{n=0}^{\infty} z^{n} \int \mid f_{m}(x)-f(x)\right) \| g\left(F^{n}(x) \mid d x \leq\left\|f_{m}-f\right\|_{1} \frac{1}{1-|z|} .\right.
\end{aligned}
$$

Therefore $\left(f_{m}, g\right)(z) \rightarrow(f, g)(z)$ as $m \rightarrow \infty$ in $|z|<1$, and

$$
\begin{aligned}
\left(f_{m}, g\right)(z) & =\sum_{|w| \leq m} C_{w}\left(1_{\langle w\rangle}, g\right)(z)=\sum_{|w| \leq m} C_{w} s_{g}^{\langle w\rangle}(z) \\
& =\sum_{k=1}^{m} \sum_{|w|=k} C_{w}\left\{\sum_{n=0}^{k-1} z^{n} \eta(w[1, n]) \int_{F^{n}\langle w\rangle} g(x) d x+z^{k} \eta(w) s_{g}^{F^{k}\langle w\rangle}(z)\right\} .
\end{aligned}
$$

Note that for a word $w$ with $|w|=k$ and $k \geq n$

$$
\left|\eta(w[1, n]) \int_{F^{n}\langle w\rangle} g(x) d x\right| \leq C e^{-(\xi-\varepsilon) k}
$$

for some constant $C>0$. Thus we get for $v^{\prime}>v$

$$
\begin{gathered}
\left|\sum_{k=1}^{\infty} \sum_{|w|=k} C_{w} \sum_{n=1}^{k-1} z^{n} \eta(w[1, n]) \int_{F^{n}\langle w\rangle} g(x) d x\right| \\
\leq C \sum_{k=1}^{\infty} \sum_{n=1}^{k-1}|z|^{n} e^{-(\xi-\varepsilon) k} \sum_{|w|=k}\left|C_{w}\right|
\end{gathered}
$$




$$
\begin{aligned}
& \leq C^{\prime} \sum_{n=1}^{\infty}|z|^{n} \sum_{k=n}^{\infty} e^{-(\xi-\varepsilon) k}\|f\|_{v^{\prime}} e^{\left(v^{\prime}+\varepsilon\right) k} \\
& \leq C^{\prime}\|f\|_{v^{\prime}} \sum_{n=1}^{\infty}|z|^{n} e^{-\left(\xi-v^{\prime}-2 \varepsilon\right) n}\left(1-e^{-\left(\xi-v^{\prime}-2 \varepsilon\right)}\right)^{-1}
\end{aligned}
$$

with some constant $C^{\prime}>0$. In a same way, we can also show that

$$
\sum_{k=1}^{\infty} \sum_{|w|=k}\left|C_{w^{\prime}} z^{k} \eta(w)\right| \leq C^{\prime \prime}\|f\|_{v^{\prime}} \sum_{n=1}^{\infty}|z|^{n} e^{-\left(\xi-v^{\prime}-2 \varepsilon\right) n}
$$

with some constant $C^{\prime \prime}>0$. Assume that $\operatorname{det}\left(I-\Phi_{n}\left(z_{0}\right)\right) \neq 0$ for $\left|z_{0}\right|<e^{\xi-v-\delta_{n}}$. Then since $s_{n, g}(z)=\left(I-\Phi_{n}(z)\right)^{-1} \chi_{n, g}(z)$, by Lemma 4.1 we get $\sup _{\tilde{K} \in \bigcup_{k=0}^{n} F^{k} \mathscr{A}}\left|s_{g}^{\widetilde{K}}\right| /\|g\|_{\infty}$ is bounded in some neighborhood $U$ of $z_{0}$. Therefore by Lemma 4.1 and Lemma 5.1, $s_{g} \boldsymbol{J}_{(z)}\left(z g \|_{\infty}\right.$ is also uniformly bounded in screens $\widetilde{J}$ and $U$. Therefore, by appealing to Lemma 2.2(ii), $\left(f_{m} \cdot g\right)(z)$ is uniformly bounded with respect to $m$ in $|z|<e^{\xi-v-\delta_{n}}$ except neighborhoods of the zeros of $\operatorname{det}\left(I-\Phi_{n}(z)\right)$. Since $s_{g}^{\tilde{a}}(z)$ is analytic in the domain $|z|<1, \operatorname{det}\left(I-\Phi_{n}(z) \not \equiv 0\right.$. Therefore the zeros of $\operatorname{det}\left(I-\Phi_{n}(z)\right)$ are isolated, and there exists an analytic continuation of $(f, g)(z)$ to $|z|<e^{\xi-v-\delta_{n}}$ except the zeros, and $\lim _{m \rightarrow \infty}\left(f_{m}, g\right)(z) \rightarrow(f, g)(z)$ in this domain. This shows for $|z|<e^{\xi-v-\delta_{n}}$ which is not the zero of $\operatorname{det}\left(I-\Phi_{n}(z)\right), \sup _{\|f\|_{v^{\prime}=1}} \sup _{\|g\|_{\infty}=1}|(f, g)(z)|<\infty$ for $v^{\prime}>v$.

On the other hand, assume $\operatorname{det}\left(I-\Phi_{n}\left(z_{0}\right)\right)=0$ for some $\left|z_{0}\right|<e^{\xi-v-\delta_{n}}$. There may happen that $V^{\prime}=\left\{\chi_{n, g}\left(z_{0}\right)\right\}_{g \in L^{\infty}}$ does not necessarily span all the space. Thus we must study the domain of $\Phi_{n}\left(z_{0}\right)$. The following discussions are almost the same as in [10]. To make notations simple, we take $n$ sufficiently large to satisy the following conditions. For any $\tilde{a}, \tilde{b} \in \tilde{A}$,

1. if $F^{i}(\tilde{a})=\tilde{b}$, then $i \leq n$,

2. if $F^{i}(\tilde{a})=F^{j}(\tilde{b})$ and $F^{i-1}(\tilde{a}) \neq F^{j-1}(\tilde{b})$ for some $i, j \geq 1$, then $i, j \leq n$.

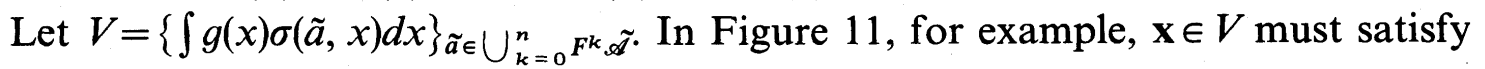

$$
\begin{gathered}
\mathbf{x}^{A C B}=\mathbf{x}^{A C}=\mathbf{x}^{A D}=\mathbf{x}^{C B D}=\mathbf{x}^{B C}=\mathbf{x}^{B D}, \\
\mathbf{x}^{C D A}=\mathbf{x}^{D C A}=\mathbf{x}^{C D(a)}=-\mathbf{x}^{C D B}=-\mathbf{x}^{D C B}=-\mathbf{x}^{C D(b)},
\end{gathered}
$$

where $C D(a)$ and $C D(b)$ are the edges corresponding to $C D$ in $\langle a\rangle$ and $\langle b\rangle$, respectively. Recall that

$$
\tilde{\chi}_{g}^{\widetilde{J}}(z)=\int g(x) \sigma(\tilde{J}, x) d x+z s_{2} \eta(a) \sum_{b \in \mathscr{A}} \sigma_{*}\left(F^{a} \widetilde{J}, b\right) \int g d x .
$$

Therefore $\tilde{\chi}_{g}^{f}\left(z_{0}\right)$ 's have relations when their screens have same inside, or when their vertex and edges coincide. From the assumption, this occurs only for $\tilde{J} \in \bigcup_{k=0}^{n-1} F^{k} \mathscr{A}$. For fixed $\left|z_{0}\right|<e^{\xi-v}$, there is an integer $m$ for which the followings hold. For any $\widetilde{\widetilde{J}} \in F^{n} \tilde{\mathscr{A}}$, $\chi_{g}^{\tilde{J}}\left(z_{0}\right)$ is an infinite series of $z_{0}$. Let us divide $\chi_{g}^{\tilde{J}}\left(z_{0}\right)=a_{g}^{\tilde{J}}+b_{g}^{\tilde{J}}$ such that $a_{g}^{\tilde{J}}$ depends only on screens $\left\{F^{k} \widetilde{J}\right\}_{0 \leq k \leq m}$ and $b_{g}^{\tilde{J}}$ depends only on screens $\left\{F^{k} \widetilde{J}\right\}_{k>m}$ and $\left|b_{g}^{\tilde{J}}\right|<\|g\|_{1} / 2 N$, where $N$ is the number of screens $\widetilde{J} \in F^{n} \mathscr{A}$. Note that the difference is estimated with $L^{1}$ norm instead of $L^{\infty}$ norm. From the assumption, $\tilde{J} \in F^{n} \mathscr{A}$ has different inside. Thus 
we can choose $g \in L^{\infty}$ with $\|g\|_{1}=1$ such that $a_{g}^{\widetilde{J}}= \pm 1 / N$. This means $\left(\chi_{g}^{\mathcal{J}}\left(z_{0}\right)\right) \boldsymbol{\gamma}_{\in F^{n} \mathscr{A}}$ spans $\mathrm{C}^{N}$. Therefore $V^{\prime} \supset V$.

On the other hand, let $\mathbf{x}=\Phi_{n}\left(z_{0}\right) \mathbf{x}$ be an eigenvector associated with the eigenvalue 1. Note that $\Phi_{n}\left(z_{0}\right)_{\tilde{a}, \tilde{b}}$ is determined by $F^{a}(\tilde{a})$. Thus, in Figure 11 , for example,

$$
\frac{\mathbf{x}^{C A D}}{z_{0} \eta(a)}=\frac{\mathbf{x}^{A C}}{z_{0} \eta(a)}=\frac{\mathbf{x}^{A D}}{z_{0} \eta(a)}=\frac{\mathbf{x}^{C B D}}{z_{0} \eta(b)}=\frac{\mathbf{x}^{B C}}{z_{0} \eta(b)}=\frac{\mathbf{x}^{C D}}{z_{0} \eta(b)} .
$$

From the assumption, when $\mathrm{x}^{\tilde{a}}$, s have relations, $F(\tilde{a}) \in \bigcup_{k=0}^{n} F^{k} \tilde{\mathscr{A}}$. This shows

$$
\mathrm{x} \in \Phi_{n}\left(z_{0}\right) V=V+\left(I-\Phi_{n}\left(z_{0}\right)\right) V .
$$

We divide an eigenvector corresponding to the eigenvalue 1 in $\mathbf{x}=\mathbf{x}_{1}+\left(I-\Phi_{n}\left(z_{0}\right)\right) \mathbf{x}_{2}$ $\left(\mathbf{x}_{1}, \mathbf{x}_{2} \in V\right)$. If there exists some $\mathbf{x}$ such that $\left(I-\Phi_{n}(z)\right)^{-1} \mathbf{x}_{1}$ is unbounded as $z \rightarrow z_{0}$, then there exists $g \in L^{\infty}$ such that $\mathbf{x}_{1}=\chi_{n, g}\left(z_{0}\right)$ and $s_{g}(z)=\left(I-\Phi_{n}(z)\right)^{-1} \chi_{n, g}(z)$ has non removable singularity at $z=z_{0}$. On the other hand, assume that $\left(I-\Phi_{n}(z)\right)^{-1} \mathbf{x}_{1}$ is bounded as $z \rightarrow z_{0}$ for any eigenvector $\mathbf{x}$ corresponding to the eigenvalue 1 . By $E$, we denote the generalized eigenspace corresponding to the eigenvalue 1 , and by $\operatorname{proj}_{E}$ we denote the projection to $E$. Then for sufficiently small $r>0$, it holds

$$
\operatorname{proj}_{E} \mathbf{y}=\int_{\left|z-z_{0}\right|=r}\left(I-\Phi_{n}(z)\right)^{-1} \mathbf{y} d z .
$$

Therefore, since $\left(I-\Phi_{n}\left(z_{0}\right)\right) \mathbf{x}_{1}=0$, we get

$$
\mathbf{x}=\operatorname{proj}_{E} \mathbf{x}=\operatorname{proj}_{E}\left(I-\Phi_{n}\left(z_{0}\right)\right) \mathbf{x}_{2} .
$$

Hence,

$$
\left(I-\Phi_{n}\left(z_{0}\right)\right)^{2} \operatorname{proj}_{E} \mathbf{x}_{2}=0 .
$$

Therefore, there exists $g \in L^{\infty}$ such that $\mathbf{x}_{2}=\chi_{n, g}\left(z_{0}\right)$, and $\mathbf{x}_{2}$ has non-zero component to the generalized eigenspace corresponding to the eigenvalue 1 . This shows $s_{g}(z)$ has also non removable singularity at $z=z_{0}$.

Therefore, there exists $\tilde{K} \in \bigcup_{k=0}^{n} F^{k} \tilde{\mathscr{A}}$ such that $s_{g}^{\tilde{K}}$ has a singularity at $z_{0}$ for some $g \in L^{\infty}$. Then by Lemma 3.2, there exists some polygon $J$ such that $s_{g}^{J}(z)=\left(1_{J}, g\right)(z)$ has a singularity at $z_{0}$. This proves the lemma.

Since $(f, g)(z)=\int(I-z P)^{-1} f(x) g(x) d x$ in $|z|<1$ and $\delta_{n} \rightarrow 0$ as $n \rightarrow \infty$, taking analytic continuation, we get:

THEOREM 5.1. Assume that $F$ is expanding and $\xi>v$. Take arbitrary $\varepsilon>0$, then there exists $n_{0}>0$ such that for $n \geq n_{0}$ and for $|z|<e^{\xi-v-\varepsilon}, z^{-1}$ is an eigenvalue of $P$ restricted to $\mathscr{B}$ if and only if $\operatorname{det}\left(I-\Phi_{n}(z)\right)=0$.

Proof. Let $\left|z_{0}\right|<e^{\xi-v-\delta_{n}}$ and $\operatorname{det}\left(I-\Phi_{n}\left(z_{0}\right)\right) \neq 0$. Then by Lemma 5.5, for any $f \in \mathscr{B}$ and $g \in L^{\infty}, \int(I-z P)^{-1} f(x) g(x) d x=(f, g)(z)$ is analytic in a neighborhood of $z_{0}$. This shows $z_{0}$ belongs to the resolvent of $P$. On the other hand, let $\left|z_{0}\right|<e^{\xi-v-\delta_{n}}$ and 
$\operatorname{det}\left(I-\Phi_{n}\left(z_{0}\right)\right)=0$. Then there exists $\tilde{a} \in \tilde{\mathscr{A}}$ such that $s_{g}^{\tilde{a}}(z)$ has a singularity at $z_{0}$. Therefore by Lemma 3.2, there exists a polygon $J$ such that $s_{g}^{J}(z)=\int(I-z P)^{-1} 1_{J}(x) g(x) d x$ has singularity at $z_{0}$. This shows $z_{0}$ is an eigenvalue of $P$ and $1_{J}$ is a corresponding eigenfunction. This proves the theorem.

\section{The proof of Theorem B.}

Now we will discuss the eigenvalues on the unit circle. We will proceed our argument along [7]. We denote the $L^{1}$ norm by $\|\cdot\|_{1}$.

LEMMA 6.1. For any $\varepsilon>0$, there exists a constant $M>0$ such that for any convex polygon $J \subset\langle w\rangle$ with some word $w \in \mathscr{W}$, there exists a decomposition

$$
1_{J}(x)=\sum_{u \in \mathscr{W}} C_{u}^{J} 1_{\langle u\rangle}(x),
$$

where $C_{u}^{J}= \pm 1$ or 0 , and $\#\left\{u:|u|=n, C_{u}^{J} \neq 0\right\}<M e^{(v+\varepsilon)(n-|w|)}$.

Proof. Using inclusion-exclusion formula, set

$$
\begin{aligned}
1_{J}(x)=1_{\langle w\rangle}(x)-\sum_{\substack{a_{1} \in \mathscr{A} \\
\left\langle w a_{1}\right\rangle \cap J^{c} \neq \varnothing}} & {\left[1_{\left\langle w a_{1}\right\rangle}(x)-\sum_{\substack{a_{2} \in \mathscr{A} \\
\left\langle w a_{1} a_{2}\right\rangle \cap J^{\circ} \neq \varnothing}}\left[1_{\left\langle w a_{1} a_{2}\right\rangle}(x)\right.\right.} \\
& \left.-\sum_{\substack{a_{3} \in \mathscr{A} \\
\left\langle w a_{1} a_{2} a_{3}\right\rangle \cap J^{c} \neq \varnothing}}\left[1_{\left\langle w a_{1} a_{2} a_{3}\right\rangle}(x)-\cdots\right]\right],
\end{aligned}
$$

where $J^{c}$ is the complement of $J$. Applying the definition of $v$ to each $F^{w}(\langle w a\rangle \cap J)$, for any $\varepsilon>0$ there exists a constant $C$ such that the number of words $u \in \mathscr{W}(|u|=n)$ for which $\langle w u\rangle$ intersect $F^{w}(\langle w\rangle \cap J)$ is bounded by $C e^{(v+\varepsilon) n}$. Note that for any word $w \in \mathscr{W}$, the number of words $w u a \in \mathscr{W}(a \in \mathscr{A})$ is at most $\# \mathscr{A}$. This proves the lemma.

Lemma 6.2. Assume that $\xi>v$. Then for each $v^{\prime}>v$, there exist $m \geq 1, \alpha>0$, and $0<\beta<1$ such that for all $f \in \mathscr{B}$

$$
\left\|P^{m} f\right\|_{v^{\prime}} \leq \alpha\|f\|_{1}+\beta\|f\|_{v^{\prime}} .
$$

Proof. For $f \in \mathscr{B}$, we take a decomposition $f=\sum_{w} C_{w} 1_{\langle w\rangle}$ such that

$$
\sum_{n=1}^{\infty} e^{-v^{\prime} n} \sum_{|w|=n}\left|C_{w}\right|<2\|f\|_{v^{\prime}}
$$

Take $\varepsilon>0$ sufficiently small such that $\xi-\varepsilon>v^{\prime}>v+\varepsilon$, and fix it. Then for any word $w$ $(|w| \geq m)$, considering $F^{|w|-m}(\langle w\rangle)$ and using Lemma 6.1, we get 


$$
\begin{aligned}
\left\|P^{m} 1_{\langle w\rangle}\right\|_{v^{\prime}} & =\left\|\eta(w[1, m]) 1_{F^{m}\langle w\rangle}\right\|_{v^{\prime}}=\eta(w[1, m])\left\|\sum_{u} C_{u}^{F^{m}\langle w\rangle} 1_{\langle u\rangle}\right\|_{v^{\prime}} \\
& =\eta(w[1, m]) \sum_{n} e^{-v^{\prime} n} \sum_{|u|=n}\left|C_{u}^{F m\langle w\rangle}\right|\left\langle\eta(w[1, m]) \sum_{n=|w|-m}^{\infty} e^{-v^{\prime} n} M e^{(v+\varepsilon)(n-|w|+m)}\right. \\
& =\eta(w[1, m]) M\left(1-e^{v+\varepsilon-v^{\prime}}\right)^{-1} e^{-v^{\prime}(|w|-m)}=\frac{M^{\prime}}{2} \eta(w[1, m]) e^{-v^{\prime}(|w|-m)}
\end{aligned}
$$

where $M^{\prime}=2 M /\left(1-e^{v+\varepsilon-v^{\prime}}\right)$. Now we choose $m$ sufficiently large such that, for any word $w \in \mathscr{W}, \eta(w[1, m])<e^{-(\xi-\varepsilon) m} / M^{\prime}$. Then we get

$$
\text { (21) }<\frac{1}{2} e^{-\left(\xi-v^{\prime}-\varepsilon\right) m} e^{-v^{\prime}|w|} .
$$

Therefore, setting $\beta=e^{-\left(\xi-v^{\prime}-\varepsilon\right) m}<1$, we get

$$
\begin{array}{r}
\left\|\sum_{|w| \geq m} C_{w} P^{m} 1_{\langle w\rangle}\right\|_{v^{\prime}} \leq \sum_{|w| \geq m}\left|C_{w}\right|\left\|P^{m} 1_{\langle w\rangle}\right\|_{v^{\prime}} \\
\leq \frac{1}{2} \beta \sum_{|w| \geq m}\left|C_{w}\right| e^{-v^{\prime}|w|} \leq \beta\|f\|_{v^{\prime}} .
\end{array}
$$

On the other hand, there exists a constant $C>0$ such that for any word $|w| \leq m$, we have a decomposition

$$
P^{m} 1_{\langle w\rangle}(x)=\sum C_{J w} 1_{J w}(x),
$$

where $\#\left\{C_{J^{w}} \neq 0\right\} \leq(\# \mathscr{A})^{m}$ and $\left|C_{J^{w}}\right|<C$, with some polygons $J^{w} \subset\left\langle a^{J^{w}}\right\rangle\left(a^{J^{w}} \in \mathscr{A}\right)$. Since

$$
\left\|1_{J w}\right\|_{v^{\prime}}=\sum_{n} e^{-v^{\prime} n} \sum_{|u|=n}\left|C_{u}^{J^{w}}\right| \leq \sum_{n} e^{-v^{\prime} n} M e^{(v+\varepsilon) n}=M^{\prime}
$$

we get

$$
\begin{aligned}
\left\|\sum_{|w|=1}^{m-1} C_{w} P^{m} 1_{\langle w\rangle}\right\| & \leq \sum_{v^{\prime}}^{m-1}\left|C_{w}\right|\left\|\sum_{J} C_{J w} 1_{J w}\right\|_{v^{\prime}} \\
& \leq \sum_{|w|=1}^{m-1}\left|C_{w}\right|(\# \mathscr{A})^{m} C\left\|1_{J w}\right\|_{v^{\prime}} \leq \sum_{|w|=1}^{m-1}\left|C_{w}\right|(\# \mathscr{A})^{m} C M^{\prime} \\
& \leq \sum_{|w|=1}^{m-1}\left|C_{w}\right| \text { Lebes }(\langle w\rangle) \delta^{-1}(\# \mathscr{A})^{m} C M^{\prime},
\end{aligned}
$$

where $\delta=\min _{1 \leq|w| \leq m-1}\{\operatorname{Lebes}(\langle w\rangle)\}>0$. Take $\alpha=\delta^{-1}(\# \mathscr{A})^{m} C M^{\prime}>0$. Then we get

$$
\left\|\sum_{|w|=1}^{m-1} C_{w} P^{m} 1_{\langle w\rangle}\right\|_{v^{\prime}} \leq \alpha\|f\|_{1} .
$$

Combining (22) and (23), we get the proof. 
THEOREM 6.1. Assume that $F$ is expanding and $\xi>v$. For $f \in L^{1}, \sum_{k=0}^{n-1} P^{k} f / n$ converges to some $f^{*} \in \mathscr{B}$ in $L^{1}$, and $P f^{*}=f^{*}$.

Proof. We can prove this theorem just in a same way as in [7]. Put for $f \in \mathscr{B}$, $\mathscr{C}=\left\{P^{m n} f\right\}_{n=0}^{\infty}$. Then by Lemma 6.2 , we get $\left\|P^{m n} f\right\|_{v^{\prime}}<\alpha /(1-\beta)\|f\|_{1}+\beta^{n}\|f\|_{v^{\prime}}$. Therefore we can choose

$$
P^{m n} f=\sum_{w \in \mathscr{W}} C_{w}^{n} 1_{\langle w\rangle}, \quad \sum_{k=1}^{\infty} e^{-v^{\prime} k} \sum_{|w|=k}\left|C_{w}^{n}\right|<\alpha /(1-\beta)\|f\|_{1}+\beta^{n}\|f\|_{v^{\prime}} .
$$

Then for any $\varepsilon>0$, there exists $M$ which is independent of $n$ such that

$$
\sum_{k \geq M} e^{-\xi k} \sum_{|w|=k}\left|C_{w}^{n}\right|<\varepsilon
$$

Now we consider some order in $\mathscr{A}$ and extend it naturally to $\mathscr{W}$. Let $\mathscr{D}$ be any infinite subset of $\mathscr{C}$. Choose an infinite sequence $\left\{n_{k}^{1}\right\}_{k=1}^{\infty}$ such that $\sum_{w \in \mathscr{W}} C_{w_{k}}^{n_{k}^{1}} 1_{\langle w\rangle} \in \mathscr{D}$ and $\left\{C_{w_{1}}^{n_{k}^{1}}\right\}$ converges as $k \rightarrow \infty$, where $w_{1}$ is the first word in the order. Then we take a subsequence $\left\{n_{k}^{2}\right\}_{k=1}^{\infty}$ of $\left\{n_{k}^{1}\right\}_{k=1}^{\infty}$ such that $\left\{C_{w_{2}}^{n_{k}^{2}}\right\}$ converges as $k \rightarrow \infty$, where $w_{2}$ is the second word, and so on. Thus by diagonal method, we can choose a sequence $\left\{n_{k}^{k}\right\}_{k=1}^{\infty}$ and there exist limits $\lim _{k \rightarrow \infty} C_{w}^{n_{k}^{k}}=C_{w}$ for all words $w \in \mathscr{W}$. Therefore by (24), it follows $\sum_{w \in \mathscr{W}}$ $C_{w} 1_{\langle w\rangle} \in L^{1}$. This shows $\mathscr{C}$ is relatively compact in $L^{1}$. Since $\left\{P^{n} f\right\}_{n=0}^{\infty} \subset \bigcup_{k=0}^{m-1} P^{k} \mathscr{C}$, $\left\{P^{n} f\right\}_{n=0}^{\infty}$ is also relatively compact. By Mazur's theorem $\left\{\sum_{k=0}^{n-1} P^{k} f / n\right\}_{n=1}^{\infty}$ is also relatively compact. Hence by Kakutani-Yosida theorem, for $f \in L^{1}$ there exists $f^{*}=Q f=\lim _{n \rightarrow \infty} \sum_{k=0}^{n-1} P^{k} f / n$ in $L^{1}$ and $P f^{*}=f^{*}$. By Lemma 6.2, $\|Q f\|_{v^{\prime}} \leq \alpha /(1-$ $\beta)\|f\|_{1}$. Note that any step function belongs to $\mathscr{B}$ and $\{\langle a\rangle: a \in \mathscr{A}\}$ is generating partition because $F$ is expanding. Thus $\mathscr{B}$ is dense in $L^{1}$. Therefore there exists a sequence $f_{n} \in \mathscr{B}$ such that $f_{n} \rightarrow f$ in $L^{1}$. Now take $v^{\prime \prime}$ such that $v^{\prime}>v^{\prime \prime}>v$ and apply Lemma 6.2 for $v^{\prime \prime}$. Then, since $Q$ is contracting, there exists some $C>0$ such that $\left\|Q f_{n}\right\|_{v^{\prime \prime}}<C\|f\|_{1}$. Choose $\left\{D_{w}^{n}\right\}$ such that

$$
Q f_{n}=\sum_{w \in \mathscr{W}} D_{w}^{n} 1_{\langle w\rangle}, \quad \sum_{k=1}^{\infty} e^{-v^{\prime \prime} k} \sum_{|w|=k}\left|D_{w}^{n}\right|<C\|f\|_{1} .
$$

Then for any $\varepsilon>0$, there exists $M>0$ which is independent of $n$ such that

$$
\sum_{k \geq M} e^{-v^{\prime \prime} k} \sum_{|w|=k}\left|D_{w}^{n}\right|<\varepsilon
$$

Therefore, just as we proved the relative compactness of $\mathscr{C}$, by diagonal method we can select $\left\{C_{w}\right\}_{w \in W}$ and

$$
Q f=\sum_{w \in W} C_{w} 1_{\langle w\rangle}, \quad \sum_{k=1}^{\infty} e^{-v^{\prime \prime} k} \sum_{|w|=k}\left|C_{w}\right|<\infty
$$

This proves the theorem. 
COROllary 6.1. Assume that $F$ is expanding and $\xi>v$. The eigenfunctions of the Perron-Frobenius operator $P$ in $L^{1}$ associated with eigenvalues modulus 1 belong to $\mathscr{B}$.

Proof. In the proof of Theorem 6.1, take $P_{\gamma} f=\gamma^{-1} P f$ instead of $P$ where $|\gamma|=1$. Suppose that $P_{\gamma} f=f$, that is $P f=\gamma f$. Then since $1 / n \sum_{k=0}^{n-1} P_{\gamma}^{k} f=f$, we get $f^{*}=$ $\lim _{n \rightarrow \infty} 1 / n \sum_{k=0}^{n-1} P_{\gamma}^{k} f=f \in \mathscr{B}$. This completes the proof.

Corollary 6.2. Assume that $F$ is expanding and $\xi>v$. There exists an invariant probability measure $\mu$, whose density function belongs to $\mathscr{B}$.

Proof. Since $P$ is a positive operator, the proof directly follows from Theorem 6.1 .

\section{References}

[1] V. Baladi and G. Keller, Zeta functions and transfer operators for piecewise monotone transformations, Comm. Math. Phys. 127 (1990), 459-478.

[2] M. L. Blank, Small perturbations of chaotic dynamical systems, Russian Math. Surveys 44 (1989), $1-33$.

[ 3] W. Feller, An Introduction to Probability Theory and Its Applications, John Wiley (1957).

[ 4 ] F. Hofbauer and G. KelLeR, Zeta functions and transfer-operators for piecewise linear transformations, J. Reine Angew. Math. 352 (1984), 100-113.

[ 5] T. Kato, Perturbation Thoery for Linear Operators, Grundlehren Math. Wiss. 132 (1966), Springer.

[6] G. KelLer, Ergodicité et mesures invariantes pour les transformations dilatantes par morceaux d'une région bornée du plan, C. R. Acad. Sci. Paris 289 (1979), 625-627.

[7] A. LASOtA and J. A. Yorke, On the existence of invariant measures for piecewise monotonic transformations, Trans. Amer. Math. Soc. 186 (1973), 481-488.

[ 8 ] M. MoRI, On the decay of correlation for piecewise monotonic mappings I, Tokyo J. Math. 8 (1985), 389-414.

[9] M. MoRI, On the decay of correlation for piecewise monotonic mappings II, Tokyo J. Math. 9 (1986), 135-161.

[10] M. MORI, Fredholm determinant for piecewise linear transformations, Osaka J. Math. 27(1990), 81-116.

[11] M. MoRI, Fredholm determinant for piecewise monotonic transformations, Osaka J. Math. 29 (1992), 497-529.

[12] M. MORI, Zeta functions and Perron-Frobenius operator of piecewise linear transformations on $\mathbf{R}^{k}$, Tokyo J. Math. 18 (1995), 401-416.

[13] M. MoRI, Fredholm determinant for higher dimensional piecewise linear transformations, preparing.

Present Address:

Department of Mathematics, College of Humanities and Sciences, Nihon University, Sakura-Josui, Setagaya-kU, Tokyo, 156-8550 JaPAN. 